ESAIM: COCV 21 (2015) 958-988

DOI: $10.1051 / \mathrm{cocv} / 2015027$
ESAIM: Control, Optimisation and Calculus of Variations

www.esaim-cocv.org

\title{
CUT TIME IN SUB-RIEMANNIAN PROBLEM ON ENGEL GROUP *
}

\author{
A.A. Ardentov ${ }^{1}$ and Yu.L. SachKov ${ }^{1}$
}

\begin{abstract}
The left-invariant sub-Riemannian problem on the Engel group is considered. The problem gives the nilpotent approximation to generic rank two sub-Riemannian problems on four-dimensional manifolds. The global optimality of extremal trajectories is studied via geometric control theory. The global diffeomorphic structure of the exponential mapping is described. As a consequence, the cut time is proved to be equal to the first Maxwell time corresponding to discrete symmetries of the exponential mapping.
\end{abstract}

Mathematics Subject Classification. 22E25, 58E25.

Received August 28, 2014. Revised March 23, 2015.

Published online June 30, 2015.

\section{INTRODUCTION}

This paper continues the study of the left-invariant sub-Riemannian problem on the Engel group started in $[6,7]$. This problem is the simplest rank 2 sub-Riemannian problem on a 4-dimensional space: it provides a nilpotent approximation to a generic sub-Riemannian problem of such kind near a generic point.

A sub-Riemannian (SR) structure on a smooth manifold $M$ is a vector distribution

$$
\Delta=\left\{\Delta_{q} \subset T_{q} M \mid q \in M\right\} \subset T M
$$

with a scalar product in $\Delta$ :

$$
g=\left\{g_{q}-\text { scalar product in } \Delta_{q} \mid q \in M\right\} .
$$

The subspaces $\Delta_{q} \subset T_{q} M$ and the scalar product $g_{q}: \Delta_{q} \times \Delta_{q} \rightarrow \mathbb{R}$ depend smoothly on a point $q \in M$. The dimension of the subspaces $\Delta_{q}$ is constant ( $\operatorname{dim} \Delta_{q}$ is called the rank of the distribution $\Delta$ ).

A Lipschitz curve $q:\left[0, t_{1}\right] \rightarrow M$ is horizontal if $\dot{q}(t) \in \Delta_{q(t)}$ for almost all $t \in\left[0, t_{1}\right]$. The length of a horizontal curve is $l=\int_{0}^{t_{1}} g(\dot{q}(t), \dot{q}(t))^{1 / 2} \mathrm{~d} t$. The sub-Riemannian distance $d\left(q_{0}, q_{1}\right)$ between points $q_{0}, q_{1} \in M$ is the infimum of lengths of horizontal curves that connect $q_{0}$ to $q_{1}$. A horizontal curve $q(t), t \in\left[0, t_{1}\right]$, is a (length) minimizer if it has a minimum possible length among all horizontal curves that connect the points $q(0)$ and $q\left(t_{1}\right)$. Description

\footnotetext{
Keywords and phrases. Sub-Riemannian geometry, optimal control, Engel group, Lie algebra, Maxwell time, cut time, exponential mapping, Euler's elastica.

* This research was partially supported by the Grant of the Russian Federation for the State Support of Researchers (Agreement No. 14.B25.31.0029)

1 Program Systems Institute of RAS, Pereslavl-Zalessky 152020, Russia. aaa@pereslavl.ru; yusachkov@gmail.com
} 
of minimizers is one of important problems of sub-Riemannian geometry. The most efficient approach to this problem is given by geometric control theory $[3,4,18]$, it consists of the following steps:

1) proof of existence of minimizers,

2) description of SR geodesics (i.e., curves whose small arcs are minimizers),

3) selection of minimizers among geodesics.

Step 1 is straightforward. If $M$ is connected and $\Delta$ is bracket generating, i.e., $\operatorname{Lie}_{q} \Delta=T_{q} M, \quad \forall q \in M$, then any points $q_{0}, q_{1} \in M$ can be connected one to another by a horizontal curve (Rashevsky-Chow theorem). If additionally the point $q_{1}$ is sufficiently close to $q_{0}$, or if the SR distance is complete, or if $\Delta$ and $g$ are left-invariant on a Lie group $M$, then $q_{0}$ can be connected with $q_{1}$ by a minimizer (Filippov theorem).

Step 2 is performed via application of Pontryagin maximum principle (PMP), which states that any geodesic (thus any minimizer) is a projection of a trajectory of a certain Hamiltonian system on the cotangent bundle $T^{*} M$. So the second step reduces to the study of integrability of the Hamiltonian system of PMP and efficient parameterization of trajectories of this system.

Step 3 is the hardest one. Local optimality of geodesics (i.e., optimality w.r.t. sufficiently close geodesics) is studied via conjugate points estimates. For the study of global optimality in problems with a big symmetry group, one can often obtain bounds (or explicit description) of cut time via the study of symmetries and global structure of the exponential mapping. We suggest the following detailing of Step 3 first applied in [5] and further developed in $[21,24-32]$ :

3.1) Discrete and continuous symmetries of the exponential mapping are found;

3.2) Maxwell points corresponding to the symmetries are found (i.e., points where several geodesics obtained one from another by a symmetry meet one another). These points (and their preimage via exponential mapping) form the Maxwell strata in the image (resp., in the preimage) of the exponential mapping. Along each geodesic, the first Maxwell time corresponding to the symmetries (i.e., the time when the geodesic meets a Maxwell strata) is found;

3.3) One proves that for any geodesic the first conjugate time is greater or equal to the first Maxwell time corresponding to the symmetries. Here the homotopy invariance of Maslov index (number of conjugate points on a geodesic) can be applied [1];

3.4) One considers restriction of the exponential mapping to the subdomains cut out in preimage and image of this mapping by the Maxwell strata corresponding to symmetries, and proves that this restriction is a diffeomorphism via Hadamard global diffeomorphism theorem [19];

3.5) On the basis of the global structure of the exponential mapping thus described, it is often possible to prove that the cut time along a geodesic (i.e., time when it loses its global optimality) is equal to the first Maxwell time corresponding to symmetries. Moreover, in this way one proves that for any terminal point in a subdomain in the image of the exponential mapping, there exists a unique minimizer which can be computed by inverting the exponential mapping in the subdomain;

3.6) Finally, for systems with big symmetry group one can construct the full optimal synthesis, and numerical algorithms and software for computation of optimal trajectories with given boundary conditions.

So far, the approach described has been applied in full just to several problems: SR problem in the flat Martinet case [5], SR problems on SO (3) and SL (2) with the Killing metric [11], SR problem on SE (2) [21,30,31], Euler elastic problem $[28,29,32]$. There are partial results on the nilpotent SR problem with the growth vector $(2,3,5)[24-27]$ and $\mathrm{SR}$ problem on $\mathrm{SH}(2)[12,13]$.

For the SR problem on the Engel group, Step 1, Step 2 and Steps 3.1, 3.2 are performed in [6] while 3.3 is done in [7]. The aim of this paper is to perform Steps 3.4, 3.5. We recall the results previously obtained in the next section.

The sub-Riemannian problem on the Engel group is a left-invariant problem on a Lie group. Such problems receive significant attention in geometric control since they provide very symmetric models which can often be studied explicitly in great detail. For left-invariant SR problems on Lie groups, one can often describe optimal 
synthesis, the structure of spheres, cut and conjugate loci. This information can give insight for general problems, where such a detailed study is much more complicated.

Left-invariant SR problems on 3D and 4D Lie groups have recently been fully classified $[2,8]$. In the 3dimensional case, optimal synthesis is known for the Heisenberg group [34], for SO (3) and SL (2) with the Killing metric $[9,11]$ and for SE (2) $[21,30,31]$. This work continues a detailed study of the simplest 4-dimensional case.

\section{Previously obtained Results}

In this section we recall results on the SR problem on the Engel group obtained previously in works $[6,7]$.

\subsection{Problem statement}

The Engel group is the 4-dimensional Lie group represented by matrices as follows:

$$
M=\left\{\left(\begin{array}{cccc}
1 & b & c & d \\
0 & 1 & a & a^{2} / 2 \\
0 & 0 & 1 & a \\
0 & 0 & 0 & 1
\end{array}\right) \mid a, b, c, d \in \mathbb{R}\right\}
$$

It is a 4-dimensional nilpotent Lie group, connected and simply connected (see an explanation of the name "Engel" for this group in [22], Sect. 6.11).

The Lie algebra of the Engel group is the four-dimensional nilpotent Lie algebra $L=\operatorname{span}\left(X_{1}, X_{2}, X_{3}, X_{4}\right)$ with the multiplication table

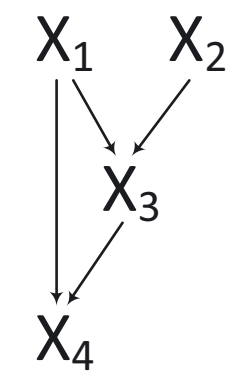

$$
\begin{aligned}
& {\left[X_{1}, X_{2}\right]=X_{3},} \\
& {\left[X_{1}, X_{3}\right]=X_{4},} \\
& {\left[X_{2}, X_{3}\right]=\left[X_{1}, X_{4}\right]=\left[X_{2}, X_{4}\right]=0 .}
\end{aligned}
$$

Thus it has graduation

$$
\begin{aligned}
& L=L_{1} \oplus L_{2} \oplus L_{3}, \\
& L_{1}=\operatorname{span}\left(X_{1}, X_{2}\right), \quad L_{2}=\mathbb{R} X_{3}, \quad L_{3}=\mathbb{R} X_{4}, \\
& {\left[L_{i}, L_{j}\right]=L_{i+j}, \quad L_{k}=\{0\} \text { for } k \geq 4,}
\end{aligned}
$$

and the Engel group is a Carnot group [22].

We consider the sub-Riemannian problem on the Engel group $M$ for the left-invariant sub-Riemannian structure generated by the orthonormal frame $X_{1}, X_{2}$ :

$$
\begin{aligned}
& \dot{q}=u_{1} X_{1}(q)+u_{2} X_{2}(q), \quad q \in M, \quad\left(u_{1}, u_{2}\right) \in \mathbb{R}^{2}, \\
& q(0)=q_{0}, \quad q\left(t_{1}\right)=q_{1}, \\
& l=\int_{0}^{t_{1}} \sqrt{u_{1}^{2}+u_{2}^{2}} \mathrm{~d} t \rightarrow \min .
\end{aligned}
$$


In appropriate coordinates $q=(x, y, z, v)$ on the Engel group $M \cong \mathbb{R}^{4}$, the problem is stated as follows:

$$
\begin{aligned}
& \dot{q}=\left(\begin{array}{c}
\dot{x} \\
\dot{y} \\
\dot{z} \\
\dot{v}
\end{array}\right)=u_{1}\left(\begin{array}{c}
1 \\
0 \\
-y / 2 \\
0
\end{array}\right)+u_{2}\left(\begin{array}{c}
0 \\
1 \\
x / 2 \\
\left(x^{2}+y^{2}\right) / 2
\end{array}\right), \quad q=(x, y, z, v) \in M=\mathbb{R}^{4}, \quad\left(u_{1}, u_{2}\right) \in \mathbb{R}^{2}, \\
& q(0)=q_{0}=\left(x_{0}, y_{0}, z_{0}, v_{0}\right), \quad q\left(t_{1}\right)=q_{1}=\left(x_{1}, y_{1}, z_{1}, v_{1}\right), \\
& l=\int_{0}^{t_{1}} \sqrt{u_{1}^{2}+u_{2}^{2}} \mathrm{~d} t \rightarrow \min .
\end{aligned}
$$

By virtue of the multiplication table (2.1) for the vector fields of the orthonormal frame

$$
X_{1}=\frac{\partial}{\partial x}-\frac{y}{2} \frac{\partial}{\partial z}, \quad X_{2}=\frac{\partial}{\partial y}+\frac{x}{2} \frac{\partial}{\partial z}+\frac{x^{2}+y^{2}}{2} \frac{\partial}{\partial v}
$$

and their Lie brackets

$$
X_{3}=\left[X_{1}, X_{2}\right]=\frac{\partial}{\partial z}+x \frac{\partial}{\partial v}, \quad X_{4}=\left[X_{1}, X_{3}\right]=\frac{\partial}{\partial v},
$$

system (2.2) is completely controllable, i.e., any points $q_{0}, q_{1} \in \mathbb{R}^{4}$ can be connected by its trajectory.

Since the problem is invariant under left shifts on the Engel group, we can assume that the initial point is the identity $q_{0}=\left(x_{0}, y_{0}, z_{0}, v_{0}\right)=(0,0,0,0)$.

\subsection{Parameterization of geodesics}

Existence of optimal solutions of problem (2.2)-(2.4) is implied by Filippov theorem [3]. By the CauchySchwarz inequality, it follows that sub-Riemannian length minimization problem (2.4) is equivalent to the action minimization problem:

$$
\int_{0}^{t_{1}} \frac{u_{1}^{2}+u_{2}^{2}}{2} \mathrm{~d} t \rightarrow \min
$$

with fixed terminal time $t_{1}$. Pontryagin maximum principle $[3,23]$ was applied to the resulting optimal control problem (2.2), (2.3), (2.5) in [6].

A sub-Riemannian geodesic can be normal or abnormal, or both. For the SR problem on the Engel group, each abnormal geodesic is simultaneously normal (see [6]), thus in the sequel we consider only normal geodesics.

Normal geodesics are projections $q_{t}=\pi\left(\lambda_{t}\right)$ via the canonical projection $\pi: T^{*} M \rightarrow M$ of solutions to the Hamiltonian system

$$
\dot{\lambda}=\overrightarrow{\mathrm{H}}(\lambda), \quad \lambda \in T^{*} M,
$$

with the Hamiltonian function $H=\frac{1}{2}\left(h_{1}^{2}+h_{2}^{2}\right)$. Here and below $h_{i}(\lambda)=\left\langle\lambda, X_{i}(q)\right\rangle, \lambda \in T^{*} M, i=1, \ldots, 4$, are Hamiltonians that correspond to the left-invariant frame and are linear on fibers of the cotangent bundle $T^{*} M$.

Arclength parameterized geodesics (i.e., with velocity $\left.g\left(\dot{q}_{t}, \dot{q}_{t}\right) \equiv 1\right)$ are projections of extremals $\lambda_{t}$ lying on the level surface $\left\{\lambda \in T^{*} M \mid H(\lambda)=1 / 2\right\}$.

Introduce coordinates $(\theta, c, \alpha)$ on the level surface $\left\{\lambda \in T^{*} M \mid H=1 / 2\right\}$ by the following formulas:

$$
h_{1}=\cos (\theta+\pi / 2), \quad h_{2}=\sin (\theta+\pi / 2), \quad h_{3}=c, \quad h_{4}=\alpha .
$$

On this surface the normal Hamiltonian system (2.6) takes the following form:

$$
\begin{aligned}
& \dot{\theta}=c, \quad \dot{c}=-\alpha \sin \theta, \quad \dot{\alpha}=0, \\
& \dot{q}=\cos \theta X_{1}(q)+\sin \theta X_{2}(q), \quad q(0)=q_{0} .
\end{aligned}
$$



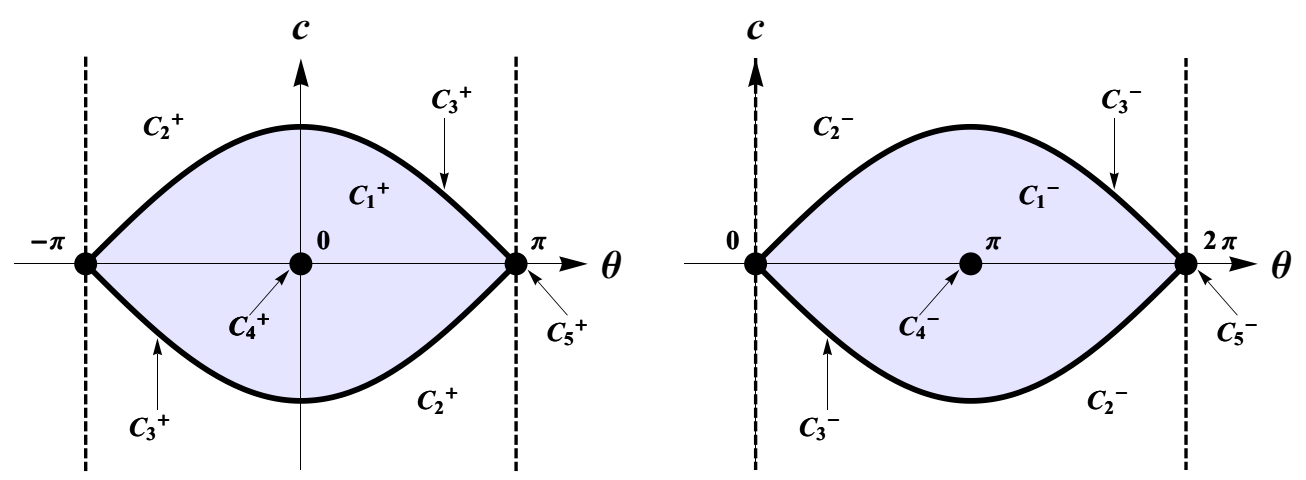

FIGURE 1. Stratification of $C$ for $\alpha>0$ and for $\alpha<0$.

The family of all normal extremals is parameterized by points of the phase cylinder of pendulum

$$
C=\left\{\lambda \in T_{q_{0}}^{*} M \mid H(\lambda)=1 / 2\right\}=\left\{(\theta, c, \alpha) \mid \theta \in S^{1}, c, \alpha \in \mathbb{R}\right\},
$$

and is given by the exponential mapping

$$
\begin{aligned}
\operatorname{Exp}: N & =C \times \mathbb{R}_{+} \rightarrow M, \\
\operatorname{Exp}(\lambda, t) & =q_{t}=\left(x_{t}, y_{t}, z_{t}, v_{t}\right) .
\end{aligned}
$$

The energy integral of pendulum (2.7) is given by $E=\frac{c^{2}}{2}-\alpha \cos \theta$. The cylinder $C$ has the following stratification corresponding to the particular type of trajectories of the pendulum:

$$
\begin{aligned}
C & =\cup_{i=1}^{7} C_{i}, \quad C_{i} \cap C_{j}=\emptyset, i \neq j, \quad \lambda=(\theta, c, \alpha), \\
C_{1} & =\{\lambda \in C \mid \alpha \neq 0, E \in(-|\alpha|,|\alpha|)\}, \\
C_{2} & =\{\lambda \in C \mid \alpha \neq 0, E \in(|\alpha|,+\infty)\}, \\
C_{3} & =\{\lambda \in C|\alpha \neq 0, E=| \alpha \mid, c \neq 0\}, \\
C_{4} & =\{\lambda \in C|\alpha \neq 0, E=-| \alpha \mid\}, \\
C_{5} & =\{\lambda \in C|\alpha \neq 0, E=| \alpha \mid, c=0\}, \\
C_{6} & =\{\lambda \in C \mid \alpha=0, c \neq 0\}, \\
C_{7} & =\{\lambda \in C \mid \alpha=c=0\} .
\end{aligned}
$$

Further, the sets $C_{i}, i=1, \ldots, 5$, are divided into subsets determined by the sign of $\alpha$ (see Fig. 1):

$$
C_{i}^{+}=C_{i} \cap\{\alpha>0\}, \quad C_{i}^{-}=C_{i} \cap\{\alpha<0\}, \quad i=1, \ldots, 5 .
$$

In order to parameterize extremal trajectories, coordinates $(\varphi, k, \alpha)$ in the domains $C_{1}$ and $C_{2}$ were introduced in $[6]$ in the following way.

In the domain $C_{1}^{+}$

$$
\begin{aligned}
k & =\sqrt{\frac{E+\alpha}{2 \alpha}}=\sqrt{\frac{c^{2}}{4 \alpha}+\sin ^{2} \frac{\theta}{2}} \in(0,1), \\
\sin \frac{\theta}{2} & =k \operatorname{sn}(\sqrt{\alpha} \varphi), \quad \cos \frac{\theta}{2}=\operatorname{dn}(\sqrt{\alpha} \varphi), \quad \frac{c}{2}=k \sqrt{\alpha} \operatorname{cn}(\sqrt{\alpha} \varphi), \quad \varphi \in[0,4 K(k)] .
\end{aligned}
$$


In the domain $C_{2}^{+}$

$$
\begin{aligned}
k & =\sqrt{\frac{2 \alpha}{E+\alpha}}=\frac{1}{\sqrt{\frac{c^{2}}{4 \alpha}+\sin ^{2} \frac{\theta}{2}}} \in(0,1), \\
\sin \frac{\theta}{2} & =\operatorname{sgn} c \operatorname{sn} \frac{\sqrt{\alpha} \varphi}{k}, \quad \cos \frac{\theta}{2}=\operatorname{cn} \frac{\sqrt{\alpha} \varphi}{k}, \quad \frac{c}{2}=\operatorname{sgn} c \frac{\sqrt{\alpha}}{k} \operatorname{dn} \frac{\sqrt{\alpha} \varphi}{k}, \quad \varphi \in[0,2 k K(k)] .
\end{aligned}
$$

Here and below dn, sn, cn are Jacobi elliptic functions depending on modulus $k, K(k)$ is the complete elliptic integral of the first kind [35].

In the domains $C_{1}^{-}, C_{2}^{-}$the coordinates $\varphi$ and $k$ are defined as follows:

$$
\varphi(\theta, c, \alpha)=\varphi(\theta-\pi, c,-\alpha), \quad k(\theta, c, \alpha)=k(\theta-\pi, c,-\alpha) .
$$

Immediate differentiation shows that system $(2.7)$ rectifies in the coordinates $(\varphi, k, \alpha)$ :

$$
\dot{\varphi}=1, \quad \dot{k}=0, \quad \dot{\alpha}=0 .
$$

In terms of these coordinates, geodesics $q_{t}=\operatorname{Exp}(\lambda, t)$ with $\lambda=(\theta, c, \alpha) \in \bigcup_{i=1}^{3} C_{i}$ and $\alpha=1$ are parameterized as follows.

If $\lambda \in C_{1}$, then

$$
\begin{aligned}
x_{t}= & 2 k\left(\operatorname{cn} \varphi_{t}-\operatorname{cn} \varphi\right), \\
y_{t}= & 2\left(\mathrm{E}\left(\varphi_{t}\right)-\mathrm{E}(\varphi)\right)-t, \\
z_{t}= & 2 k\left(\operatorname{sn} \varphi_{t} \operatorname{dn} \varphi_{t}-\operatorname{sn} \varphi \operatorname{dn} \varphi-\frac{y_{t}}{2}\left(\operatorname{cn} \varphi_{t}+\operatorname{cn} \varphi\right)\right), \\
v_{t}= & \frac{y_{t}^{3}}{6}+2 k^{2} y_{t} \operatorname{cn}^{2} \varphi-4 k^{2} \operatorname{cn} \varphi\left(\operatorname{sn} \varphi_{t} \operatorname{dn} \varphi_{t}-\operatorname{sn} \varphi \operatorname{dn} \varphi\right) \\
& +2 k^{2}\left(\frac{2}{3} \operatorname{cn} \varphi_{t} \operatorname{dn} \varphi_{t} \operatorname{sn} \varphi_{t}-\frac{2}{3} \operatorname{cn} \varphi \operatorname{dn} \varphi \operatorname{sn} \varphi+\frac{1-k^{2}}{3 k^{2}} t+\frac{2 k^{2}-1}{3 k^{2}}\left(\mathrm{E}\left(\varphi_{t}\right)-\mathrm{E}(\varphi)\right)\right) .
\end{aligned}
$$

Here and below $\mathrm{E}(\varphi)=\int_{0}^{\varphi} \mathrm{dn}^{2} t \mathrm{~d} t=E(\operatorname{am}(\varphi), k)$ is the Jacobi epsilon function and $E(u, k)$ is incomplete elliptic integral of the second kind. The Jacobi amplitude $\operatorname{am}(\varphi)$ is the inverse function of the incomplete elliptic integral of the first kind: $F(\operatorname{am}(\varphi))=\varphi$.

If $\lambda \in C_{2}$, then

$$
\begin{aligned}
x_{t}= & \frac{2 \operatorname{sgn} c}{k}\left(\operatorname{dn} \psi_{t}-\operatorname{dn} \psi\right), \\
y_{t}= & \frac{k^{2}-2}{k^{2}} t+\frac{2}{k}\left(\mathrm{E}\left(\psi_{t}\right)-\mathrm{E}(\psi)\right), \\
z_{t}= & -\frac{x_{t} y_{t}}{2}-\frac{2 \operatorname{sgn} c \operatorname{dn} \psi}{k} y_{t}+2 \operatorname{sgn} c\left(\operatorname{cn} \psi_{t} \operatorname{sn} \psi_{t}-\operatorname{cn} \psi \operatorname{sn} \psi\right), \\
v_{t}= & \frac{4}{k}\left(\frac{1}{3} \operatorname{cn} \psi_{t} \operatorname{dn} \psi_{t} \operatorname{sn} \psi_{t}-\frac{1}{3} \operatorname{cn} \psi \operatorname{dn} \psi \operatorname{sn} \psi-\frac{1-k^{2}}{3 k^{3}} t-\frac{k^{2}-2}{6 k^{2}}\left(\mathrm{E}\left(\psi_{t}\right)-\mathrm{E}(\psi)\right)\right) \\
& +\frac{y_{t}^{3}}{6}+\frac{2 y_{t}}{k^{2}} \operatorname{dn}^{2} \psi-\frac{4}{k} \operatorname{dn} \psi\left(\operatorname{cn} \psi_{t} \operatorname{sn} \psi_{t}-\operatorname{cn} \psi \operatorname{sn} \psi\right), \\
\psi= & \frac{\varphi}{k}, \quad \psi_{t}=\psi+\frac{t}{k} .
\end{aligned}
$$


If $\lambda \in C_{3}$, then

$$
\begin{aligned}
x_{t} & =2 \operatorname{sgn} c\left(\frac{1}{\cosh \varphi_{t}}-\frac{1}{\cosh \varphi}\right), \\
y_{t} & =2\left(\tanh \varphi_{t}-\tanh \varphi\right)-t \\
z_{t} & =-\frac{x_{t} y_{t}}{2}-\frac{2 \operatorname{sgn} c}{\cosh \varphi} y_{t}+2 \operatorname{sgn} c\left(\frac{\tanh \varphi_{t}}{\cosh \varphi_{t}}-\frac{\tanh \varphi}{\cosh \varphi}\right), \\
v_{t} & =\frac{2}{3}\left(\tanh \varphi_{t}-\tanh \varphi+2 \frac{\tanh \varphi_{t}}{\cosh ^{2} \varphi_{t}}-2 \frac{\tanh \varphi}{\cosh ^{2} \varphi}\right)+\frac{y_{t}^{3}}{6}+\frac{2 y_{t}}{\cosh ^{2} \varphi}-\frac{4}{\cosh \varphi}\left(\frac{\tanh \varphi_{t}}{\cosh \varphi_{t}}-\frac{\tanh \varphi}{\cosh \varphi}\right) .
\end{aligned}
$$

Parameterization of geodesics for $\lambda \in \bigcup_{i=1}^{3} C_{i}$ and arbitrary $\alpha \neq 0$ is obtained from the above parameterization for $\alpha=1$ via the following symmetries of the Hamiltonian system: dilations

$$
\begin{aligned}
& \delta_{\mu}:(\theta, c, \alpha, t, x, y, z, v) \mapsto\left(\theta, c / \mu, \alpha / \mu^{2}, \mu t, \mu x, \mu y, \mu^{2} z, \mu^{3} v\right), \quad \mu>0, \\
& \delta_{\mu}:(\varphi, k, \alpha) \mapsto\left(\mu \varphi, k, \alpha / \mu^{2}\right),
\end{aligned}
$$

and reflection

$$
\begin{aligned}
& (\theta, c, \alpha, t, x, y, z, v) \mapsto(\theta-\pi, c,-\alpha, t,-x,-y, z,-v), \\
& (\varphi, k, \alpha) \mapsto(\varphi, k,-\alpha) .
\end{aligned}
$$

In the remaining cases $\lambda \in \bigcup_{i=4}^{7} C_{i}$ geodesics are parameterized by elementary functions as follows.

$\lambda \in C_{4}$ :

$$
x_{t}=0, \quad y_{t}=t \operatorname{sgn} \alpha, \quad z_{t}=0, \quad v_{t}=\frac{t^{3}}{6} \operatorname{sgn} \alpha .
$$

$\lambda \in C_{5}:$

$$
x_{t}=0, \quad y_{t}=-t \operatorname{sgn} \alpha, \quad z_{t}=0, \quad v_{t}=-\frac{t^{3}}{6} \operatorname{sgn} \alpha .
$$

$\lambda \in C_{6}:$

$$
\begin{aligned}
x_{t} & =\frac{\cos (c t+\theta)-\cos \theta}{c}, & y_{t} & =\frac{\sin (c t+\theta)-\sin \theta}{c}, \\
z_{t} & =\frac{c t-\sin (c t)}{2 c^{2}}, & v_{t} & =\frac{3 \cos \theta-2 c t \sin \theta-4 \cos (c t+\theta)+\cos (2 c t+\theta)}{4 c^{3}} .
\end{aligned}
$$

$\lambda \in C_{7}:$

$$
x_{t}=-t \sin \theta, \quad y_{t}=t \cos \theta, \quad z_{t}=0, \quad v_{t}=\frac{t^{3}}{6} \cos \theta .
$$

Projections of geodesics to the plane $(x, y)$ are Euler elasticae (stationary configurations of planar elastic rod with fixed endpoints and tangents at endpoints) $[15,20,28,29,32]$ : inflexional ones for $\lambda \in C_{1}$, non-inflexional ones for $\lambda \in C_{2}$, critical ones for $\lambda \in C_{3}$, straight lines for $\lambda \in C_{4} \cup C_{5} \cup C_{7}$, and circles for $\lambda \in C_{6}$.

\subsection{Symmetries of exponential mapping}

A pair of mappings

$$
s: N \rightarrow N, \quad s: M \rightarrow M
$$

is called a symmetry of the exponential mapping if it commutes with this mapping:

$$
s \circ \operatorname{Exp}(\lambda, t)=\operatorname{Exp} \circ s(\lambda, t), \quad(\lambda, t) \in N .
$$




\subsection{Dilations}

A one-parameter group of symmetries of the exponential mapping is formed by dilations

$$
\begin{aligned}
\delta_{\mu}:(\theta, c, \alpha, t) & \mapsto\left(\theta, c / \mu, \alpha / \mu^{2}, \mu t\right), \\
\delta_{\mu}:(x, y, z, v) & \mapsto\left(\mu x, \mu y, \mu^{2} z, \mu^{3} v\right), \quad \mu>0 .
\end{aligned}
$$

Dilations act on Euler elastiae as homotheties.

\subsection{Reflections}

The following mappings $\varepsilon^{i}: C \rightarrow C$ preserve the field of directions of the vertical part of the Hamiltonian vector field $\overrightarrow{\mathrm{H}}_{v}=c \frac{\partial}{\partial \theta}-\alpha \sin \theta \frac{\partial}{\partial c} \in \operatorname{Vec}(C)$ :

$$
\begin{array}{lll}
\varepsilon^{1}:(\theta, c, \alpha) & \mapsto(\theta,-c, \alpha), & \varepsilon^{2}:(\theta, c, \alpha) \mapsto(-\theta, c, \alpha), \\
\varepsilon^{3}:(\theta, c, \alpha) \mapsto(-\theta,-c, \alpha), & \varepsilon^{4}:(\theta, c, \alpha) \mapsto(\theta+\pi, c,-\alpha), \\
\varepsilon^{5}:(\theta, c, \alpha) \mapsto(\theta+\pi,-c,-\alpha), & & \varepsilon^{6}:(\theta, c, \alpha) \mapsto(-\theta+\pi, c,-\alpha), \\
\varepsilon^{7}:(\theta, c, \alpha) \mapsto(-\theta+\pi,-c,-\alpha) . & &
\end{array}
$$

More precisely, $\varepsilon_{*}^{i} \overrightarrow{\mathrm{H}}_{v}=\overrightarrow{\mathrm{H}}_{v}$ for $i=3,4,7$, and $\varepsilon_{*}^{i} \overrightarrow{\mathrm{H}}_{v}=-\overrightarrow{\mathrm{H}}_{v}$ for $i=1,2,5,6$. The action of reflections $\varepsilon^{i}$ is continued to symmetries of the exponential mapping as follows.

The action $\varepsilon^{i}: N \rightarrow N$ is defined as

$$
\varepsilon^{i}(\lambda, t)= \begin{cases}\left(\varepsilon^{i}(\lambda), t\right), & \text { if } \varepsilon_{*}^{i} \overrightarrow{\mathrm{H}}_{v}=\overrightarrow{\mathrm{H}}_{v}, \\ \left(\varepsilon^{i} \circ e^{t \overrightarrow{\mathrm{H}}_{v}}(\lambda), t\right), & \text { if } \varepsilon_{*}^{i} \overrightarrow{\mathrm{H}}_{v}=-\overrightarrow{\mathrm{H}}_{v} .\end{cases}
$$

The action $\varepsilon^{i}: M \rightarrow M$ is defined as

$$
\begin{aligned}
\varepsilon^{i}(q) & =\varepsilon^{i}(x, y, z, v)=q^{i}=\left(x^{i}, y^{i}, z^{i}, v^{i}\right), \\
\left(x^{1}, y^{1}, z^{1}, v^{1}\right) & =(x, y,-z, v-x z), \\
\left(x^{2}, y^{2}, z^{2}, v^{2}\right) & =(-x, y, z, v-x z), \\
\left(x^{3}, y^{3}, z^{3}, v^{3}\right) & =(-x, y,-z, v), \\
\left(x^{4}, y^{4}, z^{4}, v^{4}\right) & =(-x,-y, z,-v), \\
\left(x^{5}, y^{5}, z^{5}, v^{5}\right) & =(-x,-y,-z,-v+x z), \\
\left(x^{6}, y^{6}, z^{6}, v^{6}\right) & =(x,-y, z,-v+x z), \\
\left(x^{7}, y^{7}, z^{7}, v^{7}\right) & =(x,-y,-z,-v) .
\end{aligned}
$$

The mappings $\varepsilon^{i}$ act on endpoints of Euler elasticae as reflections in coordinate axes or in the origin.

Thus defined reflections $\varepsilon^{i}, i=1, \ldots, 7$, form a discrete group of symmetries of the exponential mapping (together with the identity mapping). We denote this group $G=\left\{\operatorname{Id}, \varepsilon^{1}, \ldots, \varepsilon^{7}\right\}$.

\subsection{Maxwell points}

A point $q_{t}$ of an extremal trajectory $q_{s}=\operatorname{Exp}(\lambda, s)$ is called a Maxwell point if there exists another extremal trajectory $\widetilde{q}_{s}=\operatorname{Exp}(\widetilde{\lambda}, s), \widetilde{q}_{s} \not \equiv q_{s}$, such that $\widetilde{q}_{t}=q_{t}$. The instant $t$ is called a Maxwell time. It is known [27] that an extremal trajectory cannot be optimal after a Maxwell time.

The main result of paper [6], given by Theorem 2.1 below, provides an upper bound of the cut time along extremal curves

$$
t_{\text {cut }}(\lambda)=\sup \{t>0 \mid \operatorname{Exp}(\lambda, s) \text { is optimal for } s \in[0, t]\} .
$$


Define the following function $t_{\mathrm{MAX}}^{1}: C \rightarrow(0,+\infty]$ :

$$
\begin{array}{ll}
\lambda \in C_{1} & \Rightarrow t_{\mathrm{MAX}}^{1}=\min \left(2 p_{z}^{1}(k), 4 K(k)\right) / \sigma, \\
\lambda \in C_{2} & \Rightarrow t_{\mathrm{MAX}}^{1}=2 k K(k) / \sigma, \\
\lambda \in C_{6} & \Rightarrow t_{\mathrm{MAX}}^{1}=2 \pi /|c|, \\
\lambda \in C_{3} \cup C_{4} \cup C_{5} \cup C_{7} & \Rightarrow t_{\mathrm{MAX}}^{1}=+\infty,
\end{array}
$$

where $\sigma=\sqrt{|\alpha|} ; K(k)=\int_{0}^{\frac{\pi}{2}} \frac{\mathrm{d} t}{\sqrt{1-k^{2} \sin ^{2} t}} ; p_{z}^{1}(k) \in(K(k), 3 K(k))$ is the first positive root of the function $f_{z}(p, k)=\operatorname{dn} p \operatorname{sn} p+(p-2 \mathrm{E}(p)) \operatorname{cn} p$.

Theorem 2.1 ([6], Thm. 3). For any $\lambda \in C$

$$
t_{\text {cut }}(\lambda) \leq t_{\mathrm{MAX}}^{1}(\lambda)
$$

Proposition 2.2. The function $t_{\mathrm{MAX}}^{1}: C \rightarrow(0,+\infty]$ has the following invariant properties:

1) $t_{\mathrm{MAX}}^{1}(\lambda)$ depends only on the values of $E$ and $|\alpha|$;

2) $t_{\mathrm{MAX}}^{1}(\lambda)$ is an integral of the vector field $\overrightarrow{\mathrm{H}}_{v}$;

3) $t_{\mathrm{MAX}}^{1}(\lambda)$ is invariant w.r.t. reflections: if $\lambda \in C, \lambda^{i}=\varepsilon^{i}(\lambda) \in C$, then $t_{\mathrm{MAX}}^{1}\left(\lambda^{i}\right)=t_{\mathrm{MAX}}^{1}(\lambda)$;

4) $t_{\mathrm{MAX}}^{1}$ respects the action of dilations: if $\lambda \in C, \lambda_{\mu}=\delta_{\mu}(\lambda)$, then $t_{\mathrm{MAX}}^{1}\left(\lambda_{\mu}\right)=\mu t_{\mathrm{MAX}}^{1}(\lambda)$.

Proof.

1) We denote by $\sqcup$ the union of disjoint sets. Notice first that the decomposition

$$
C=C_{1} \sqcup C_{2} \sqcup C_{35} \sqcup C_{4} \sqcup C_{6} \sqcup C_{7}
$$

with $C_{35}=C_{3} \cup C_{5}=\{\lambda \in C|\alpha \neq 0, E=| \alpha \mid\}$ is determined only by the functions $E$ and $|\alpha|$, see definitions (2.8)-(2.14). Thus it remains to show that restriction of $t_{\mathrm{MAX}}^{1}$ to each of the subsets in decomposition (2.36) depends only on $E$ and $|\alpha|$.

If $\lambda \in C_{1}$, then $k=\sqrt{\frac{E+|\alpha|}{2|\alpha|}}$, thus $k=k(E,|\alpha|)$, so $t_{\mathrm{MAX}}^{1}=t_{\mathrm{MAX}}^{1}(E,|\alpha|)$.

The case $\lambda \in C_{2}$ is similar to the case $\lambda \in C_{1}$.

If $\lambda \in C_{35} \cup C_{4} \cup C_{7}$, then $t_{\mathrm{MAX}}^{1}=+\infty=t_{\mathrm{MAX}}^{1}(E,|\alpha|)$.

Finally, if $\lambda \in C_{6}$, then $t_{\mathrm{MAX}}^{1}=\frac{2 \pi}{|c|}=\frac{\sqrt{2} \pi}{\sqrt{E}}$.

2) Since $E$ and $\alpha$ are integrals of the vector field $\overrightarrow{\mathrm{H}}_{v}$, then $t_{\mathrm{MAX}}^{1}=t_{\mathrm{MAX}}^{1}(E,|\alpha|)$ is an integral of $\overrightarrow{\mathrm{H}}_{v}$ as well.

3) Let $\lambda \in C, \varepsilon^{i}(\lambda)=\lambda^{i} \in C$. Since $E\left(\lambda^{i}\right)=E(\lambda), \alpha\left(\lambda^{i}\right)= \pm \alpha(\lambda)$ and $t_{\mathrm{MAX}}^{1}=t_{\mathrm{MAX}}^{1}(E,|\alpha|)$, then $t_{\mathrm{MAX}}^{1}\left(\lambda^{i}\right)=$ $t_{\operatorname{MAX}}^{1}(\lambda)$.

4) Let $\lambda \in C$ and $\lambda_{\mu}=\delta_{\mu}(\lambda), \mu>0$. Since we have $E\left(\lambda_{\mu}\right)=\frac{1}{\mu^{2}} E(\lambda)$ and $\alpha\left(\lambda_{\mu}\right)=\frac{1}{\mu^{2}} \alpha(\lambda)$, then $\delta_{\mu}\left(C_{i}\right)=$ $C_{i}, i=1, \ldots, 7$, and $k\left(\lambda_{\mu}\right)=k(\lambda)$. Then it follows from the definition of the function $t_{\mathrm{MAX}}^{1}$ that $t_{\mathrm{MAX}}^{1}\left(\lambda_{\mu}\right)=$ $\mu \cdot t_{\mathrm{MAX}}^{1}(\lambda)$ for $\lambda \in C_{i}$ and each $i=1, \ldots, 7$.

\subsection{Conjugate points}

A point $q_{t}=\operatorname{Exp}(\lambda, t)$ is called a conjugate point for $q_{0}$ if $\nu=(\lambda, t)$ is a critical point of the exponential mapping and that is why $q_{t}$ is the corresponding critical value:

$$
d_{\nu} \operatorname{Exp}: T_{\nu} N \rightarrow T_{q_{t}} M \text { is degenerate. }
$$

The instant $t$ is called a conjugate time along the extremal trajectory $q_{s}=\operatorname{Exp}(\lambda, s), s \geq 0$. 
The first conjugate time along a trajectory $\operatorname{Exp}(\lambda, s)$ is denoted by

$$
t_{\text {conj }}^{1}(\lambda)=\min \{t>0 \mid t \text { is a conjugate time along } \operatorname{Exp}(\lambda, s), s \geq 0\} .
$$

The trajectory $\operatorname{Exp}(\lambda, s)$ loses its local optimality at the instant $t=t_{\text {conj }}^{1}(\lambda)$ (see [3]).

The following lower bound on the first conjugate time is the main result of work [7].

Theorem $2.3([7])$. For any $\lambda \in C$

$$
t_{\mathrm{conj}}^{1}(\lambda) \geq t_{\mathrm{MAX}}^{1}(\lambda)
$$

\section{Decompositions in PREImage And image of EXPonential MAPPing}

In this section we describe decomposition (3.6) in the image, and decomposition (3.12) in the preimage of the exponential mapping, which will be proved to be diffeomorphic via the exponential mapping in Theorem 4.20.

\subsection{Decomposition in $M$}

Let $\widehat{M}=M \backslash\left\{q_{0}\right\}$, then $M=\widehat{M} \sqcup\left\{q_{0}\right\}$. Further, we denote the subset containing the Maxwell strata MAX $^{1}$ and $\mathrm{MAX}^{2}$ :

$$
M^{\prime}=\{q \in \widehat{M} \mid x z=0\}
$$

and its complement

$$
\widetilde{M}=\{q \in \widehat{M} \mid x z \neq 0\}
$$

then

$$
\widehat{M}=\widetilde{M} \sqcup M^{\prime} .
$$

Denote the connected components of the set $\widetilde{M}$ :

$$
\begin{aligned}
& M_{1}=\{q \in M \mid x<0, z>0\}, \\
& M_{2}=\{q \in M \mid x<0, z<0\}, \\
& M_{3}=\{q \in M \mid x>0, z<0\}, \\
& M_{4}=\{q \in M \mid x>0, z>0\},
\end{aligned}
$$

so that

$$
\widetilde{M}=\bigsqcup_{i=1}^{4} M_{i} .
$$

This decomposition agrees with the action of reflections and dilations as described in the following statement.

\section{Proposition 3.1.}

1) Reflections $\varepsilon^{j} \in G$ permute the domains $M_{i}$ according to Table 1 .

2) Dilations $\delta_{\mu}, \mu>0$, preserve the domains $M_{i}$.

Proof. Follows immediately from the definitions of the actions of reflections $\varepsilon^{j}: M \rightarrow M$, see (2.23)-(2.30), and dilations $\delta_{\mu}: M \rightarrow M$, see $(2.22)$. 
TABLE 1. Action of the reflections $\varepsilon^{j}$ on the domains $M_{i}$.

\begin{tabular}{cccc}
\hline$I d, \varepsilon^{6}$ & $\varepsilon^{1}, \varepsilon^{7}$ & $\varepsilon^{2}, \varepsilon^{4}$ & $\varepsilon^{3}, \varepsilon^{5}$ \\
\hline$M_{1}$ & $M_{2}$ & $M_{4}$ & $M_{3}$ \\
$M_{2}$ & $M_{1}$ & $M_{3}$ & $M_{4}$ \\
$M_{3}$ & $M_{4}$ & $M_{2}$ & $M_{1}$ \\
$M_{4}$ & $M_{3}$ & $M_{1}$ & $M_{2}$ \\
\hline
\end{tabular}

\subsection{Decomposition in $N$}

Denote the subset in preimage of the exponential mapping that corresponds to all potentially optimal geodesics:

$$
\widehat{N}=\left\{(\lambda, t) \in N \mid t \leq t_{\text {MAX }}^{1}(\lambda)\right\}
$$

If $(\lambda, t) \in N \backslash \widehat{N}$, then the geodesic $\operatorname{Exp}(\lambda, s), s \in[0, t]$, is non-optimal. We decompose the set $\widehat{N}$ into subsets corresponding to the subsets of the set $\widehat{M}$ (Subsect. 3.1), the proof of this correspondence will be given in Subsection 3.3. Let

$$
\begin{aligned}
& N^{\prime}=\left\{(\lambda, t) \in N \mid t=t_{\mathrm{MAX}}^{1}(\lambda) \text { or } c_{t / 2} \sin \theta_{t / 2}=0\right\}, \\
& \widetilde{N}=\left\{(\lambda, t) \in N \mid t<t_{\mathrm{MAX}}^{1}(\lambda), c_{t / 2} \sin \theta_{t / 2} \neq 0\right\}
\end{aligned}
$$

then

$$
\widehat{N}=\tilde{N} \sqcup N^{\prime} .
$$

The following sets will play an important role in the description of the global structure of the exponential mapping:

$$
\begin{aligned}
& D_{1}=\left\{(\lambda, t) \in N \mid t \in\left(0, t_{\mathrm{MAX}}^{1}(\lambda)\right), \sin \theta_{t / 2}>0, c_{t / 2}>0\right\}, \\
& D_{2}=\left\{(\lambda, t) \in N \mid t \in\left(0, t_{\mathrm{MAX}}^{1}(\lambda)\right), \sin \theta_{t / 2}>0, c_{t / 2}<0\right\}, \\
& D_{3}=\left\{(\lambda, t) \in N \mid t \in\left(0, t_{\mathrm{MAX}}^{1}(\lambda)\right), \sin \theta_{t / 2}<0, c_{t / 2}<0\right\}, \\
& D_{4}=\left\{(\lambda, t) \in N \mid t \in\left(0, t_{\mathrm{MAX}}^{1}(\lambda)\right), \sin \theta_{t / 2}<0, c_{t / 2}>0\right\} .
\end{aligned}
$$

We have the obvious decomposition

$$
\widetilde{N}=\bigsqcup_{i=1}^{4} D_{i} .
$$

The trace of domains $D_{i}$ in the set $\{(\lambda, t) \in N \mid t=0\}$ is shown in Figure 2.

\section{Proposition 3.2.}

1) Reflections $\varepsilon^{j} \in G$ permute the sets $D_{i}$ according to Table 2 .

2) Dilations $\delta_{\mu}, \mu>0$, preserve the sets $D_{i}$.

\section{Proof.}

1) We prove only the equality $\varepsilon^{1}\left(D_{1}\right)=D_{2}$, all the rest equalities given in Table 2 are proved similarly. Let $(\lambda, t)=(\theta, c, \alpha, t) \in D_{1}$ and $\varepsilon^{1}(\lambda, t)=\left(\lambda^{1}, t\right)=\left(\theta^{1}, c^{1}, \alpha^{1}, t\right)$, we show that $\left(\lambda^{1}, t\right) \in D_{2}$.

Denote $\lambda_{t / 2}=\left(\theta_{t / 2}, c_{t / 2}, \alpha\right)=\mathrm{e}^{(t / 2) \overrightarrow{\mathrm{H}}_{v}}(\lambda)$ and $\lambda_{t / 2}^{1}=\left(\theta_{t / 2}^{1}, c_{t / 2}^{1}, \alpha^{1}\right)=\mathrm{e}^{(t / 2) \overrightarrow{\mathrm{H}}_{v}}\left(\lambda^{1}\right)$. Since $\varepsilon_{*}^{1} \overrightarrow{\mathrm{H}}_{v}=-\overrightarrow{\mathrm{H}}_{v}$, then $\lambda^{1}=\varepsilon^{1} \circ \mathrm{e}^{t \overrightarrow{\mathrm{H}}_{v}}(\lambda)$, thus

$$
\lambda_{t / 2}^{1}=\mathrm{e}^{(t / 2) \overrightarrow{\mathrm{H}}_{v}} \circ \varepsilon^{1} \circ \mathrm{e}^{t \overrightarrow{\mathrm{H}}_{v}}(\lambda)=\varepsilon^{1} \circ \mathrm{e}^{-(t / 2) \overrightarrow{\mathrm{H}}_{v}} \circ \mathrm{e}^{t \overrightarrow{\mathrm{H}}_{v}}(\lambda)=\varepsilon^{1} \circ \mathrm{e}^{(t / 2) \overrightarrow{\mathrm{H}}_{v}}(\lambda)=\varepsilon^{1}\left(\lambda_{t / 2}\right) .
$$




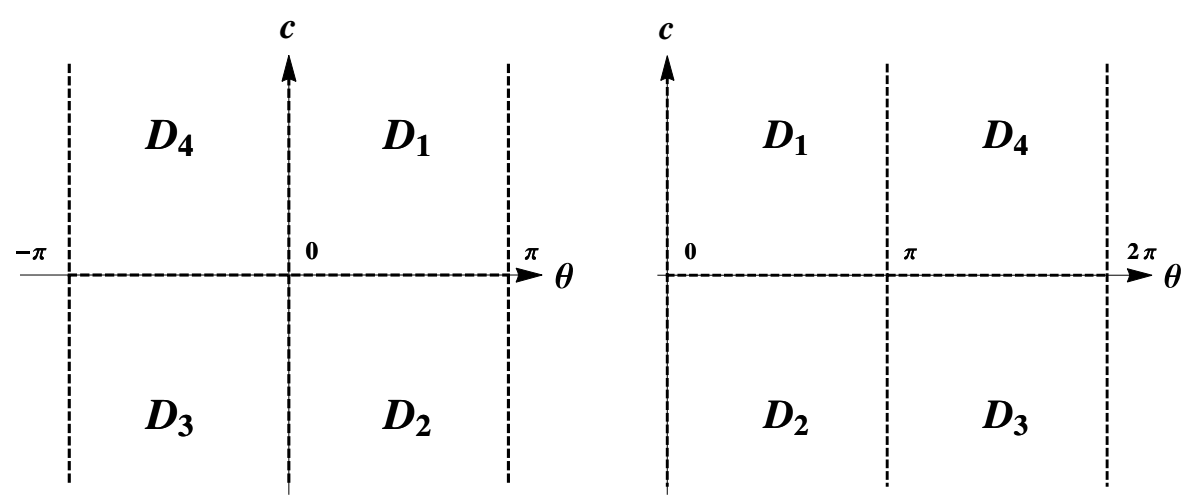

Figure 2. The trace of domains $D_{i}$ in the set $\{t=0\}$ for $\alpha \geq 0$ and for $\alpha<0$.

TABLE 2. Action of the reflections $\varepsilon^{j}$ on the domains $D_{i}$.

\begin{tabular}{cccc}
\hline$I d, \varepsilon^{6}$ & $\varepsilon^{1}, \varepsilon^{7}$ & $\varepsilon^{2}, \varepsilon^{4}$ & $\varepsilon^{3}, \varepsilon^{5}$ \\
\hline$D_{1}$ & $D_{2}$ & $D_{4}$ & $D_{3}$ \\
$D_{2}$ & $D_{1}$ & $D_{3}$ & $D_{4}$ \\
$D_{3}$ & $D_{4}$ & $D_{2}$ & $D_{1}$ \\
$D_{4}$ & $D_{3}$ & $D_{1}$ & $D_{2}$ \\
\hline
\end{tabular}

That is,

$$
\left(\theta_{t / 2}^{1}, c_{t / 2}^{1}, \alpha^{1}\right)=\left(\theta_{t / 2},-c_{t / 2}, \alpha\right)
$$

The inclusion $(\lambda, t) \in D_{1}$ means that

$$
t \in\left(0, t_{\mathrm{MAX}}^{1}(\lambda)\right), \quad \sin \theta_{t / 2}>0, \quad c_{t / 2}>0,
$$

thus $\sin \theta_{t / 2}^{1}>0, c_{t / 2}^{1}<0$. Moreover, since $t_{\mathrm{MAX}}^{1}\left(\lambda^{1}\right)=t_{\mathrm{MAX}}^{1} \circ \varepsilon^{1}(\lambda)=t_{\mathrm{MAX}}^{1}(\lambda)$ by Proposition 2.2, then $t \in\left(0, t_{\mathrm{MAX}}^{1}\left(\lambda^{1}\right)\right)$. Consequently, $\left(\lambda^{1}, t\right) \in D_{2}$.

We proved that $\varepsilon^{1}\left(D_{1}\right) \subset D_{2}$. It follows similarly that $\varepsilon^{1}\left(D_{2}\right) \subset D_{1}$. Since $\varepsilon^{1} \circ \varepsilon^{1}=$ Id on $N$, we have $\varepsilon^{1}\left(D_{1}\right)=D_{2}$.

2) Let $(\lambda, t)=(\theta, c, \alpha, t) \in N,\left(\lambda_{\mu}, t_{\mu}\right)=\delta_{\mu}(\lambda, t)=\left(\theta, c / \mu, \alpha / \mu^{2}, \mu t\right)$. Since $t_{\mathrm{MAX}}^{1}\left(\lambda_{\mu}\right)=\mu t_{\mathrm{MAX}}^{1}(\lambda)$ by Proposition 2.2, it is obvious that if $(\lambda, t) \in D_{1}$, then $\left(\lambda_{\mu}, t_{\mu}\right) \in D_{1}$. Thus $\delta_{\mu}\left(D_{1}\right) \subset D_{1}$. Since $d_{1 / \mu}=\left(\delta_{\mu}\right)^{-1}$, then $\delta_{\mu}\left(D_{1}\right)=D_{1}$. It follows similarly that $\delta_{\mu}\left(D_{i}\right)=D_{i}$ for $i=2,3,4$.

\subsection{Basic properties of exponential mapping}

In this subsection we describe some simple properties on the action of the exponential mapping on the subsets of $N$ defined in the previous subsection.

First of all, $\operatorname{Exp}(\widehat{N}) \supset \widehat{M}$ since for any point $q_{1} \in \widehat{M}$ there exists an optimal trajectory $q_{s}=\operatorname{Exp}(\lambda, s)$ such that $q_{t_{1}}=q_{1}$, thus $t_{1} \leq t_{\text {cut }}(\lambda) \leq t_{\mathrm{MAX}}^{1}(\lambda)$, i.e., $\operatorname{Exp}\left(\lambda, t_{1}\right)=q_{1}$ with $\left(\lambda, t_{1}\right) \in \widehat{N}$. However, Maxwell points in $\widehat{M}$ have several preimages in $\widehat{N}$. Moreover, the mapping $\left.\operatorname{Exp}\right|_{\widehat{N}}$ is degenerate at points $(\lambda, t)$ where $t=t_{\mathrm{MAX}}^{1}(\lambda)$ is a conjugate time along the trajectory $\operatorname{Exp}(\lambda, s)$.

In the next two propositions we show that the action of Exp is compatible with decompositions (3.7), (3.1), and (3.12), (3.6). 
Proposition 3.3. There holds the inclusion

$$
\operatorname{Exp}\left(N^{\prime}\right) \subset\{q \in M \mid x z=0\}=M^{\prime} \sqcup\left\{q_{0}\right\}
$$

Proof. The reflection $\varepsilon^{4}$ and dilations $\delta_{\mu}, \mu>0$, are symmetries of Exp and preserve the sets $N^{\prime}, M^{\prime}$ and $\left\{q_{0}\right\}$. Since $\varepsilon^{4}: \alpha \mapsto-\alpha$ and $\delta_{\mu}: \alpha \mapsto \alpha / \mu^{2}$, we can assume in the proof of inclusion (3.13) that $\alpha \in\{0,1\}$.

Let $(\lambda, t) \in N^{\prime}$ and $q_{t}=\left(x_{t}, y_{t}, z_{t}, v_{t}\right)=\operatorname{Exp}(\lambda, t)$, we show that $x_{t} z_{t}=0$.

Suppose first that $\alpha=1$, then $\lambda \in \bigcup_{i=1}^{5} C_{i}$.

Let $\lambda \in C_{1}$, then we use parameterization of extremals (2.15). Since $(\lambda, t) \in N^{\prime}$, then $c_{t / 2} \sin \theta_{t / 2}=0$ or $t=$ $t_{\mathrm{MAX}}^{1}(\lambda)$. If $c_{t / 2}=2 k \mathrm{cn} \tau=0$, then $\mathrm{cn} \tau=0$, thus $z_{t}=0$ in view of (7.3) from [6]. If $\sin \theta_{t / 2}=2 k \operatorname{sn} \tau \operatorname{dn} \tau=0$, then $x_{t}=0$ in view of (7.2) from [6]. Finally, if $t=t_{\mathrm{MAX}}^{1}(\lambda)$, then $p=p_{z}^{1}(k)$ or $p=2 K(k)$ by $(2.31)$, thus $z_{t}=0$ or $x_{t}=0$ by (7.2) and (7.3) from [6].

The case $\lambda \in C_{2} \cup C_{3}$ is considered similarly to the case $\lambda \in C_{1}$.

If $\lambda \in C_{4} \cup C_{5}$, then $x_{t}=0$ by (2.18), (2.19).

Now suppose that $\alpha=0$, thus $\lambda \in C_{6} \cup C_{7}$.

Let $\lambda \in C_{6}$, then we use parameterization of extremals (2.20). The case $c_{t / 2}=0$ is impossible. If $\sin \theta_{t / 2}=0$, then $t=2 \pi /|c|$, thus $x_{t}=0$. If $t=t_{\mathrm{MAX}}^{1}(\lambda)=2 \pi /|c|(2.33)$, then $x_{t}=0$ as well.

Finally, if $\lambda \in C_{7}$, then $z_{t}=0$ by $(2.20)$.

Proposition 3.4. For any $i=1, \ldots, 4$, we have $\operatorname{Exp}\left(D_{i}\right) \subset M_{i}$.

Proof. By virtue of the reflections $\varepsilon^{i}$ (Prop. 3.1, 3.2), the proof of this proposition reduces to the case $i=1$. So let $(\lambda, t) \in D_{1}$, we prove that $q_{t}=\operatorname{Exp}(\lambda, t) \in M_{1}$.

The reflection $\varepsilon^{6}$ and the dilations $\delta_{\mu}, \mu>0$, preserve the domains $D_{1}$ and $M_{1}$, and act on the parameter $\alpha$ as $\varepsilon^{6}: \alpha \mapsto-\alpha, \delta_{\mu}: \alpha \mapsto \frac{\alpha}{\mu^{2}}$, thus we can assume in this proof that $\alpha \in\{0,1\}$.

Since $(\lambda, t) \in D_{1}$, then $\sin \theta_{t / 2}>0, c_{t / 2}>0, t \in\left(0, t_{\mathrm{MAX}}^{1}(\lambda)\right)$.

Let $\alpha=1$, then $\lambda \in C_{1} \cup C_{2} \cup C_{3}$.

Let $\lambda \in C_{1}$. Then $\sin \theta_{t / 2}=2 k \operatorname{sn} \tau \operatorname{dn} \tau>0, c_{t / 2}=2 k \operatorname{cn} \tau>0$. Since in this case $t \in\left(0, t_{\text {MAX }}^{1}(\lambda)\right)$ and $t_{\mathrm{MAX}}^{1}(\lambda)=\min \left(2 p_{z}^{1}, 4 K\right)$, then $f_{z}(p, k)>0$ and $\operatorname{sn} p>0$. Then formulas (7.2), (7.3) from [6] imply that $x_{t}<0, z_{t}>0$, i.e., $q_{t} \in M_{1}$.

The cases $\lambda \in C_{2}$ and $\lambda \in C_{3}$ are considered similarly to the case $\lambda \in C_{1}$.

Now let $\alpha=0$, then $\lambda \in C_{6}$. Then $x_{t}=-\frac{2}{c} \sin \theta_{t / 2} \sin \frac{c t}{2}<0$ and $z_{t}=\frac{c t-\sin (c t)}{2 c^{2}}>0$, thus $q_{t} \in M_{1}$.

We proved that $\operatorname{Exp}\left(D_{1}\right) \subset M_{1}$.

Our goal is to prove that the mappings Exp: $D_{i} \rightarrow M_{i}, i=1, \ldots, 4$, are diffeomorphisms (see Cor. 4.21). This is done in Section 4 via the following Hadamard global diffeomorphism theorem.

Theorem 3.5 ([19]). Let $F: X \rightarrow Y$ be a smooth mapping between smooth manifolds of equal dimension. Let the following conditions hold:

1) $X$ is connected;

2) $Y$ is connected and simply connected;

3) $F$ is nondegenerate;

4) $F$ is proper (i.e., $F^{-1}(K) \subset X$ is compact for a compact $K \subset Y$ ).

Then $F$ is a diffeomorphism. 


\subsection{Topological properties of decompositions in $M$ and $N$}

We prove that hypotheses (1), (2) of Theorem 3.5 are verified for the mappings Exp: $D_{i} \rightarrow M_{i}$.

Definition 3.6. Let $X$ be a topological space and $f_{1}, f_{2}: X \rightarrow \mathbb{R}$. Then $f_{1} \sim f_{2}$ on a sequence $\left\{\lambda_{n}\right\} \subset X$ if $\lim _{n \rightarrow \infty} \frac{f_{1}\left(\lambda_{n}\right)}{f_{2}\left(\lambda_{n}\right)}=1$

\section{Proposition 3.7.}

1) The sets $D_{i} \subset N, i=1, \ldots, 4$, are open and connected.

2) The sets $M_{i} \subset M, i=1, \ldots, 4$, are open, connected and simply connected.

In the proof of item (1) of this proposition we need the following statement.

Proposition 3.8. The function $t_{\mathrm{MAX}}^{1}: C \rightarrow(0,+\infty]$ is continuous on the set $C \backslash C_{4}$, and is smooth on the set $C_{1}^{0} \cup C_{2}$, where $C_{1}^{0}=\left\{\lambda \in C_{1} \mid k \neq k_{0}\right\}$.

Remark 3.9. We assume in $(0,+\infty]$ the natural basis of topology:

$$
(a, b), \quad(a,+\infty], \quad 0<a<b<+\infty .
$$

Proof. Let $\lambda_{n} \rightarrow \bar{\lambda}$ as $n \rightarrow \infty$, where $\lambda_{n}, \bar{\lambda} \in C \backslash C_{4}=\left(\cup_{i=1}^{3} C_{i}\right) \cup\left(\cup_{i=5}^{7} C_{i}\right)$. We denote $t_{n}=t_{\mathrm{MAX}}^{1}\left(\lambda_{n}\right)$ and $\bar{t}=t_{\mathrm{MAX}}^{1}(\bar{\lambda})$, then prove that $t_{n} \rightarrow \bar{t}$ as $n \rightarrow+\infty$.

1. Let $\lambda_{n} \in C_{1}$, then $\bar{\lambda} \in \operatorname{cl}\left(C_{1}\right) \backslash C_{4}=C_{1} \cup C_{3} \cup C_{5} \cup C_{7}$.

1.1. Let $\bar{\lambda} \in C_{1}$. The function $\left.t_{\mathrm{MAX}}^{1}\right|_{C_{1}}=\frac{\min \left(2 p_{z}^{1}(k), 4 K(k)\right)}{\sigma}$ is continuous since for $k \in(0,1)$ the function $\min \left(p_{z}^{1}(k), 2 K(k)\right)$ is continuous (see [32], Cor. 3.1), thus $t_{n} \rightarrow \bar{t}$.

1.2. Let $\bar{\lambda} \in C_{3} \cup C_{5}$. Then $k_{n}=k\left(\lambda_{n}\right) \rightarrow 1, K\left(k_{n}\right) \rightarrow+\infty, p_{z}^{1}\left(k_{n}\right) \rightarrow+\infty, \sigma\left(\lambda_{n}\right) \rightarrow \bar{\sigma}>0$. Thus $t_{n} \rightarrow+\infty=\bar{t}$.

1.3. Let $\bar{\lambda} \in C_{7}$. Then $\alpha_{n} \rightarrow 0$. Since $\min \left(2 p_{z}^{1}(k), 4 K(k)\right)>2 K(k)>\pi$, then $t_{n} \rightarrow+\infty=\bar{t}$.

2. Let $\lambda_{n} \in C_{2}$, then $\bar{\lambda} \in \operatorname{cl}\left(C_{2}\right) \backslash C_{4}=C_{2} \cup C_{3} \cup C_{5} \cup C_{6} \cup C_{7}$.

2.1. Let $\bar{\lambda} \in C_{2}$. The function $\left.t_{\mathrm{MAx}}^{1}\right|_{C_{2}}=\frac{2 K(k) k}{\sigma}$ is continuous, thus $t_{n} \rightarrow \bar{t}$.

2.2. Let $\bar{\lambda} \in C_{3} \cup C_{5}$. This case is similar to Case 1.2 .

2.3. Let $\bar{\lambda} \in C_{6}$. Then

$$
\begin{array}{ll}
\alpha_{n}=\alpha\left(\lambda_{n}\right) \rightarrow \bar{\alpha}=0, & c_{n}=c\left(\lambda_{n}\right) \rightarrow \bar{c} \neq 0, \\
E_{n}=E\left(\lambda_{n}\right) \rightarrow \frac{\bar{c}^{2}}{2}=\bar{E} \neq 0, & k_{n}=k\left(\lambda_{n}\right)=\sqrt{\frac{2\left|\alpha_{n}\right|}{E_{n}+\left|\alpha_{n}\right|} \sim \frac{2 \sqrt{\left|\alpha_{n}\right|}}{|\bar{c}|} \rightarrow 0,} \\
t_{n}=\frac{2 K\left(k_{n}\right) k_{n}}{\sqrt{\left|\alpha_{n}\right|}} \sim 2 K(0) \cdot \frac{2}{|\bar{c}|}=\frac{2 \pi}{|\bar{c}|}=\bar{t}, &
\end{array}
$$

i.e., $t_{n} \rightarrow \bar{t}$.

2.4. Let $\bar{\lambda} \in C_{7}$. Then $\alpha_{n} \rightarrow 0, c_{n} \rightarrow 0$. Thus $E_{n} \rightarrow 0$, so $\frac{k_{n}}{\sqrt{\left|\alpha_{n}\right|}}=\sqrt{\frac{2}{E_{n}+\left|\alpha_{n}\right|}} \rightarrow+\infty$. Consequently, $t_{n}=\frac{2 K\left(k_{n}\right) k_{n}}{\sqrt{\left|\alpha_{n}\right|}} \rightarrow+\infty=\bar{t}$

3. Let $\lambda_{n} \in C_{3}$, then $\bar{\lambda} \in \operatorname{cl}\left(C_{3}\right) \backslash C_{4}=C_{3} \cup C_{5} \cup C_{7}$, and this case is similar to Cases 1.2 and 1.3.

4. Let $\lambda_{n} \in C_{5}$, then $\bar{\lambda} \in \operatorname{cl}\left(C_{5}\right)=C_{5} \cup C_{7}$, and $t_{n}=+\infty=\bar{t}$.

5. Let $\lambda_{n} \in C_{6}$, then $\bar{\lambda} \in \operatorname{cl}\left(C_{6}\right)=C_{6} \cup C_{7}$. 
5.1. Let $\bar{\lambda} \in C_{6}$. Since the function $\left.t_{\mathrm{MAX}}^{1}\right|_{C_{6}}=\frac{2 \pi}{|c|}$ is continuous, then $t_{n} \rightarrow \bar{t}$.

5.2. Let $\bar{\lambda} \in C_{7}$. Then $c_{n} \rightarrow 0$, thus $t_{n}=\frac{2 \pi}{\left|c_{n}\right|} \rightarrow+\infty=\bar{t}$.

The function $t_{\operatorname{MAX}}^{1}(\lambda)$ is smooth on $C_{1}^{0}$ since for $\lambda \in C_{1}$ we have by virtue of (2.31):

$$
\begin{aligned}
& k<k_{0} \Rightarrow t_{\mathrm{MAX}}^{1}=\frac{2 p_{z}^{1}(k)}{\sqrt{|\alpha|}} \in C^{\infty}, \\
& k>k_{0} \Rightarrow t_{\mathrm{MAX}}^{1}=\frac{4 K}{\sqrt{|\alpha|}} \in C^{\infty} .
\end{aligned}
$$

Similarly, $t_{\mathrm{MAX}}^{1}(\lambda)$ is smooth on $C_{2}$ by virtue of (2.32). The proof of Proposition 3.8 is complete.

Remark 3.10. The function $t_{\mathrm{MAX}}^{1}$ is discontinuous on $C_{4}$.

Indeed, let $\lambda_{n} \in C_{1}$ be such that $k\left(\lambda_{n}\right) \rightarrow 0$ and $\alpha\left(\lambda_{n}\right) \rightarrow \bar{\alpha} \neq 0$. Then $\lambda_{n} \rightarrow \bar{\lambda} \in C_{4}$ but

$$
t_{\mathrm{MAX}}^{1}\left(\lambda_{n}\right) \rightarrow \frac{2 p_{z}^{1}(0)}{\sqrt{|\bar{\alpha}|}}<t_{\mathrm{MAX}}^{1}(\bar{\lambda})=+\infty .
$$

Here $p=p_{z}^{1}(0)$ is the minimal positive root of the equation $f_{z}(p, 0)=\sin p-p \cos p=0$, thus $p \in(\pi, 3 \pi / 2)$.

Now we prove Proposition 3.7.

\section{Proof.}

1) Reflections $\varepsilon^{i}: N \rightarrow N$ are diffeomorphisms and permute the sets $D_{i}$, thus it is sufficient to prove that the set $D_{1}=\left\{(\lambda, t) \in N \mid \sin \theta_{t / 2}>0, c_{t / 2}>0, t<t_{\mathrm{MAX}}^{1}(\lambda)\right\}$ is open and connected.

Consider the vector field $P=\frac{t}{2}\left(c \frac{\partial}{\partial \theta}-\alpha \sin \theta \frac{\partial}{\partial c}\right) \in \operatorname{Vec}(N)$. Denote the flow of this vector field for time 1 as $e^{P} \in \operatorname{Diff}(N)$. We have

$$
\mathrm{e}^{P}(\theta, c, \alpha, t)=\mathrm{e}^{P}(\lambda, t)=\left(\mathrm{e}^{\frac{t}{2} \overrightarrow{\mathrm{H}}_{v}}(\lambda), t\right)=\left(\theta_{t / 2}, c_{t / 2}, \alpha, t\right),
$$

thus $e^{P}\left(D_{1}\right)=\widetilde{D}_{1}$, where

$$
\widetilde{D}_{1}=\left\{(\lambda, t) \in N \mid \sin \theta>0, c>0, t<t_{\mathrm{MAX}}^{1}(\lambda, t)\right\} .
$$

By Proposition 3.8, the function $\left(t-t_{\mathrm{MAX}}^{1}(\lambda)\right): N \rightarrow(0,+\infty]$ is continuous on the set $N \backslash N_{4} \supset \widetilde{D}_{1}$, thus the set $\widetilde{D}_{1}$ is open. Moreover, the domain $\widetilde{D}_{1}$ is a subgraph of the function $t_{\mathrm{MAX}}^{1}(\lambda)$ on a connected domain $\{(\theta, c, \alpha) \in C \mid \theta \in(0, \pi), c>0, \alpha \in \mathbb{R}\}$, thus $\widetilde{D}_{1}$ is connected.

We proved that $\widetilde{D}_{1}$ is open and connected, thus $D_{1}=e^{-P}\left(\widetilde{D}_{1}\right)$ is open and connected as well.

2) It is obvious from definitions (3.2)-(3.5) that the sets $M_{i}, i=1, \ldots 4$, are open, connected and simply connected.

\section{DifFEOMORPHIC PROPERTIES OF EXPONENTIAL MAPPING}

In this section we prove that restriction of the exponential mapping to the subdomains $D_{i}, M_{i}$ is a diffeomorphism.

Lemma 4.1. If $\operatorname{Exp}: D_{1} \rightarrow M_{1}$ is proper, then $\operatorname{Exp}: D_{i} \rightarrow M_{i}$ is proper for $i=2,3,4$.

Proof. Follows immediately from Propositions 3.1, 3.2. 
Lemma 4.2. The mapping Exp : $D_{1} \rightarrow M_{1}$ is proper iff there exists no sequence $\left\{\nu_{n}\right\} \subset D_{1}=\left(D_{1} \cap N_{1}\right) \cup$ $\left(D_{1} \cap N_{2}\right) \cup\left(D_{1} \cap N_{3}\right) \cup\left(D_{1} \cap N_{6}\right)$, such that $\nu_{n} \rightarrow \bar{\nu} \in \operatorname{cl}\left(D_{1}\right) \backslash D_{1}$ and $\operatorname{Exp}\left(\nu_{n}\right) \rightarrow \bar{q} \in M_{1}$.

Proof. It follows from the definition of a proper mapping that the mapping Exp : $D_{1} \rightarrow M_{1}$ is proper iff there exists no sequence $\left\{\nu_{n}\right\} \subset D_{1}$, such that $\nu_{n} \rightarrow \bar{\nu} \in \operatorname{cl}\left(D_{1}\right) \backslash D_{1}$ and $\operatorname{Exp}\left(\nu_{n}\right) \rightarrow \bar{q} \in M_{1}$.

Moreover, the definition of $D_{1}$ (3.8) gives the decomposition

$$
D_{1}=\left(D_{1} \cap N_{1}\right) \cup\left(D_{1} \cap N_{2}\right) \cup\left(D_{1} \cap N_{3}\right) \cup\left(D_{1} \cap N_{6}\right) .
$$

Let us introduce the following sets for arbitrary $\varepsilon \in(0,1)$ :

$$
S_{\varepsilon}:=\left\{\nu \in N\left|\theta_{t / 2} \in[\varepsilon, \pi-\varepsilon], c_{t / 2} \in[\varepsilon, 1 / \varepsilon],\right| \alpha \mid \leq 1 / \varepsilon, t \in[\varepsilon, 1 / \varepsilon], t_{\mathrm{MAX}}^{1}(\lambda)-t \geq \varepsilon\right\} .
$$

Lemma 4.3. The set $S_{\varepsilon}$ is compact for any $\varepsilon>0$.

Proof. Let $\left\{\nu_{n}\right\} \subset S_{\varepsilon}$ be an arbitrary sequence. To prove the lemma, we need to find a subsequence $\nu_{n_{m}}$ which tends to $\bar{\nu} \in S_{\varepsilon}$ as $m \rightarrow \infty$.

Since $\alpha, t$ are bounded on $S_{\varepsilon}$, we obtain for a subsequence that $\alpha \rightarrow \bar{\alpha}, t \rightarrow \bar{t}$ as $m \rightarrow \infty$.

Since $\theta_{t / 2}, c_{t / 2}$ are bounded on $S_{\varepsilon}$, we obtain for a subsequence $\left(\theta_{t / 2}, c_{t / 2}\right) \rightarrow(a, b)$. Moreover, we have $(\theta, c)=\Phi_{-t / 2}\left(\theta_{t / 2}, c_{t / 2}\right) \rightarrow \Phi_{-\bar{t} / 2}(a, b)=:(\bar{\theta}, \bar{c})$, where $\Phi$ is the flow of pendulum $(2.7)$. Since $\bar{\nu}=(\bar{\theta}, \bar{c}, \bar{\alpha}, \bar{t}) \in S_{\varepsilon}$ by continuity of the functions which define $S_{\varepsilon}$, we see that $S_{\varepsilon}$ is compact.

Lemma 4.4. If $K \subset D_{1}$ is compact, then there exists $\varepsilon>0$ such that $K \subset S_{\varepsilon}$.

Proof. Since the functions $\theta_{t / 2}, c_{t / 2}, \alpha, t,\left(t_{\mathrm{MAX}}^{1}-t\right)$ are continuous on $N$, these functions attain maximum and minimum on $K$.

Lemma 4.5. Let $\left\{\nu_{n}\right\} \subset D_{1}$. Then $\nu_{n} \rightarrow \bar{\nu} \in \operatorname{cl}\left(D_{1}\right) \backslash D_{1}$ iff one of the following conditions holds for $\left\{\nu_{n}\right\}$ :

1) $\theta_{t / 2} \rightarrow 0$

2) $\theta_{t / 2} \rightarrow \pi$

3) $c_{t / 2} \rightarrow 0$

4) $c_{t / 2} \rightarrow+\infty$;

5) $t \rightarrow 0$;

6) $t_{\mathrm{MAX}}^{1}(\lambda)-t \rightarrow 0$;

7) $|\alpha| \rightarrow \infty$.

Proof. Necessity. Assume the converse. Suppose for any sequence $\left\{\nu_{n}\right\} \subset D_{1}, \nu_{n} \rightarrow \bar{\nu} \in \operatorname{cl}\left(D_{1}\right) \backslash D_{1}$, that conditions (1-7) do not hold. This means that there exists $\varepsilon>0$ such that conditions

$$
\theta_{t / 2} \geq \varepsilon, \theta_{t / 2} \leq \pi-\varepsilon, \quad c_{t / 2} \geq \varepsilon, c_{t / 2} \leq 1 / \varepsilon, \quad t \geq \varepsilon, t_{\mathrm{MAX}}^{1}(\lambda)-t \geq \varepsilon, \quad|\alpha| \leq 1 / \varepsilon
$$

hold for a subsequence. It follows that $\left\{\nu_{n}\right\} \subset S_{\varepsilon}$, which is a compact subset of $D_{1}$. So $\bar{\nu} \in S_{\varepsilon} \subset D_{1}$. This contradiction proves the necessity.

Sufficiency. Assume the converse. Let for any sequence $\nu_{n} \subset D_{1}$ we have $\nu_{n} \rightarrow \bar{\nu} \in D_{1}$. Then there exists a compact set $K \supset\left\{\nu_{n}\right\}, \bar{\nu} \in K$. This means that there exists $\varepsilon>0$ such that $K \subset S_{\varepsilon}$. This contradiction proves the lemma.

Definition 4.6. Let $X$ be a topological space and $f_{1}, f_{2}: X \rightarrow \mathbb{R}$. Then $f_{1} \approx f_{2}$ on a sequence $\left\{\nu_{n}\right\} \subset X$ if $\lim _{n \rightarrow \infty} \frac{f_{1}\left(\nu_{n}\right)}{f_{2}\left(\nu_{n}\right)} \in \mathbb{R} \backslash\{0\}$.

In the next lemmas we use the parametrization of exponential mapping for the case $\lambda \in C_{6}$ (see (2.20)).

Lemma 4.7. If $\left\{\nu_{n}\right\} \subset D_{1} \cap N_{6}, \nu_{n} \rightarrow \bar{\nu} \in \operatorname{cl}\left(D_{1}\right) \backslash D_{1}$, and $\operatorname{Exp}\left(\nu_{n}\right) \rightarrow \bar{q} \in M_{1}$, then $c \rightarrow 0$ on the sequence $\left\{\nu_{n}\right\}$. 
Proof. Notice that for $\bar{\nu}=\{\bar{x}, \bar{y}, \bar{z}, \bar{v}\} \in M_{1}$ we have $\bar{x} \neq 0$. Consider all possible cases $\bar{\nu} \in \operatorname{cl} D_{1} \backslash D_{1}$ :

1) $\theta_{t / 2} \rightarrow 0 \Rightarrow \frac{c t}{2}+\theta \rightarrow 0 \Rightarrow c \rightarrow 0$ or $x=-\frac{2 \sin \left(\frac{c t}{2}+\theta\right) \sin \frac{c t}{2}}{c} \rightarrow \bar{x}=0$.

2) $\theta_{t / 2} \rightarrow \pi \Rightarrow \frac{c t}{2}+\theta \rightarrow 0 \Rightarrow c \rightarrow 0$ or $x \rightarrow \bar{x}=0$.

3) $c_{t / 2} \rightarrow 0 \Rightarrow c \rightarrow 0$.

4) $c_{t / 2} \rightarrow \infty \Rightarrow c \rightarrow \infty \Rightarrow x \rightarrow \bar{x}=0$.

5) $t \rightarrow 0 \Rightarrow c \rightarrow 0$, otherwise $x \rightarrow \bar{x}=0$.

6) $t \rightarrow \frac{2 \pi}{|c|}$. This means that $c \rightarrow 0$ or $x \rightarrow \bar{x}=0$.

Lemma 4.8. Suppose $\nu_{n} \in D_{1} \cap N_{6}$. If $c \rightarrow 0$, then $x \rightarrow 0$ or one of the functions $x, y, z$ tends to $\infty$ on the sequence $\left\{\nu_{n}\right\}$.

Proof. Consider two possible cases:

1) If $c t \rightarrow 0$, then

$$
\begin{aligned}
z & \approx \frac{(c t)^{3}}{c^{2}}=c t^{3}, \\
x^{2}+y^{2} & =\frac{2-2(\cos (c t+\theta) \cos \theta+\sin (c t+\theta) \sin \theta)}{c}=\frac{2(1-\cos (c t))}{c} \approx c t^{2} .
\end{aligned}
$$

It follows that $t \rightarrow \infty$, otherwise $x^{2} \rightarrow 0$. Then we have $z \approx\left(x^{2}+y^{2}\right) t$. This means that $z \approx t \rightarrow \infty$ or $x^{2} \rightarrow 0$.

2) If $c t \rightarrow \bar{c} \neq 0$ or $c t \rightarrow \infty$, then $c t-\sin (c t)>M>0$, thus $z \rightarrow \infty$.

In the next lemmas we use the following parametrization of exponential mapping for the case $\lambda \in C_{3}$ (see $(2.17))$ :

$$
\begin{aligned}
x= & -\frac{8 \operatorname{sgn} c \sigma \sinh p \sinh \tau}{\alpha(\cosh (2 p)+\cosh (2 \tau))}, \\
y= & \frac{2 \sigma}{\alpha}\left(\frac{2 \sinh (2 p)}{\cosh (2 p)+\cosh (2 \tau)}-p\right) \\
z= & \frac{8 \operatorname{sgn} c \cosh \tau(p \cosh p-\sinh p)}{|\alpha|(\cosh (2 p)+\cosh (2 \tau))} \\
v= & -\frac{1}{3 \alpha \sigma \cosh (p-\tau) \cosh ^{2}(p+\tau)}(6(\cosh \tau-3 \cosh (2 p+\tau)) \sinh p \\
& +2 p(6 \cosh (3 p+\tau)+p \cosh (p+\tau)(p(\cosh (2 p)+\cosh (2 \tau))-6 \sinh (2 p)))),
\end{aligned}
$$

where $\tau=\sigma\left(\varphi+\varphi_{t}\right) / 2, p=\sigma t / 2$.

Lemma 4.9. If $\left\{\nu_{n}\right\} \subset D_{1} \cap N_{3}, \nu_{n} \rightarrow \bar{\nu} \in \operatorname{cl}\left(D_{1}\right) \backslash D_{1}$, and $\operatorname{Exp}\left(\nu_{n}\right) \rightarrow \bar{q} \in M_{1}$, then $\sigma \rightarrow 0$ or $p \rightarrow \infty$ and $t \rightarrow \infty$ with $\sigma \rightarrow \bar{\sigma} \neq 0$.

Proof. Notice that for $\bar{\nu}=\{\bar{x}, \bar{y}, \bar{z}, \bar{v}\} \in M_{1}$ we have $\bar{x} \neq 0$. Consider all possible cases $\nu \rightarrow \operatorname{cl}\left(D_{1}\right) \backslash D_{1}$ :

1) $\theta_{t / 2} \rightarrow 0 \Rightarrow\left\{\begin{array}{l}\tanh \tau \rightarrow 0 \\ \cosh \tau \rightarrow 1\end{array} \Rightarrow \tau \rightarrow 0 \Rightarrow \sigma \rightarrow 0\right.$, or $x \rightarrow \bar{x}=0$, or $z \rightarrow \infty$ with $p \rightarrow \infty$. 
2) $\theta_{t / 2} \rightarrow \pi \Rightarrow\left\{\begin{array}{l}\tanh \tau \rightarrow 1 \\ \frac{1}{\cosh \tau} \rightarrow 0\end{array} \Rightarrow \tau \rightarrow \infty \Rightarrow\left\{\begin{array}{l}p \rightarrow \infty \\ \tau \rightarrow \infty\end{array}\right.\right.$ or $\sigma \rightarrow 0$.

3) $c_{t / 2} \rightarrow 0 \Rightarrow \frac{\sigma}{\cosh \tau} \rightarrow 0 \Rightarrow\left[\begin{array}{r}\sigma \rightarrow 0 \\ \cosh \tau \rightarrow \infty\end{array} \Rightarrow\left\{\begin{array}{l}p \rightarrow \infty \\ \tau \rightarrow \infty\end{array}\right.\right.$ or $\sigma \rightarrow 0$.

4) $c_{t / 2} \rightarrow \infty \Rightarrow \frac{\sigma}{\cosh \tau} \rightarrow \infty \Rightarrow \sigma \rightarrow \infty \Rightarrow x \rightarrow \bar{x}=0$

5) $t \rightarrow 0 \Rightarrow \frac{p}{\sigma} \rightarrow 0 \Rightarrow\left[\begin{array}{c}p \rightarrow 0 \\ \sigma \rightarrow \infty\end{array} \Rightarrow\left[\begin{array}{l}\sigma \rightarrow 0 \\ x \rightarrow 0\end{array} \Rightarrow \sigma \rightarrow 0\right.\right.$.

6) $t \rightarrow \infty \Rightarrow \frac{p}{\sigma} \rightarrow \infty \Rightarrow\left[\begin{array}{c}p \rightarrow \infty \\ \sigma \rightarrow 0\end{array} \Rightarrow\left\{\begin{array}{l}p \rightarrow \infty \\ \tau \rightarrow \infty\end{array}\right.\right.$ or $\sigma \rightarrow 0$

7) $|\alpha| \rightarrow \infty \Rightarrow\left[\begin{array}{l}p \rightarrow \infty, \\ \tau \rightarrow \infty,\end{array}\right.$ or $x \rightarrow \bar{x}=0$.

Lemma 4.10. Suppose $\nu_{n} \in D_{1} \cap N_{3}$. If $p \rightarrow \infty, \tau \rightarrow \infty, \sigma \rightarrow \bar{\sigma} \neq 0$, then $y \rightarrow \infty$.

Proof. Since $\frac{2 \sinh (2 p)}{\cosh (2 p)+\cosh (2 \tau)}<\infty$ for $p \rightarrow \infty$, then $y \rightarrow \infty$.

Lemma 4.11. Suppose $\nu_{n} \in D_{1} \cap N_{3}$. If $\sigma \rightarrow 0$, then one of the functions $x, y, z, v$ tends to $\infty$, otherwise $x$ or $z$ tends to 0 .

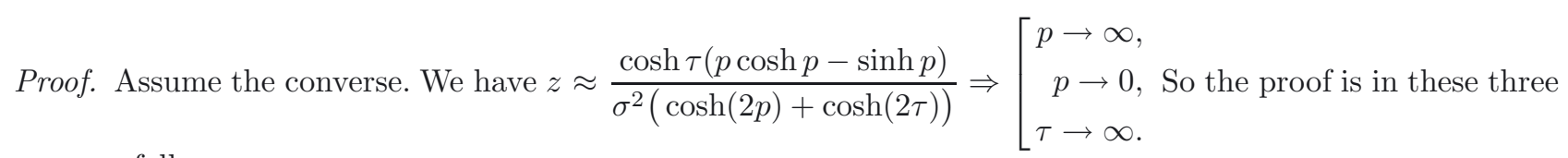
cases as follows:

1) $p \rightarrow \infty$. Then we get $y \approx \frac{1}{\sigma} p \rightarrow \infty$.

2) $p \rightarrow 0$. Consider three subcases:

2.1) $\tau \rightarrow 0$. Here we have $x \approx \frac{\tau p}{\sigma} \Rightarrow \sigma \approx \tau p$ and $y \approx \frac{p}{\sigma} \approx \frac{p}{\tau p}=\frac{1}{\tau} \rightarrow \infty$.

2.2) $\tau \rightarrow \infty$. We obtain

$$
\begin{aligned}
& x \approx \frac{p}{\sigma e^{\tau}} \Rightarrow p \approx \sigma e^{\tau}, \\
& z \approx \frac{p^{3}}{\sigma^{2} e^{\tau}} \approx \frac{p^{2}}{\sigma} \Rightarrow \sigma \approx p^{2}, \\
& y \approx \frac{1}{\sigma}\left(\frac{p}{\cosh ^{2} \tau}-p\right) \approx \frac{p}{\sigma} \approx \frac{1}{p} \rightarrow \infty .
\end{aligned}
$$

2.3) $\tau \rightarrow \bar{\tau}<\infty, \bar{\tau} \neq 0$. It follows that $x \approx \frac{p}{\sigma} \Rightarrow p \approx \sigma$, then $z \approx \frac{p^{3}}{\sigma^{2}} \approx \sigma \rightarrow 0$.

3) $\tau \rightarrow \infty, p \rightarrow \bar{p}<\infty, \bar{p} \neq 0$. We get $\frac{\sinh (2 p)}{\cosh (2 p)+\cosh (2 \tau)} \rightarrow 0 \Rightarrow y \approx \frac{\bar{p}}{\sigma} \rightarrow \infty$. 
Below in the case $\nu \in\left(D_{1} \cap N_{1}\right) \cup\left(D_{1} \cap N_{2}\right)$ we use the following notation:

$$
\begin{aligned}
& \nu \in N_{1} \Rightarrow \tau=\sigma\left(\varphi+\varphi_{t}\right) / 2, \quad p=\sigma t / 2, \\
& \nu \in N_{2} \Rightarrow \tau=\sigma\left(\varphi+\varphi_{t}\right) /(2 k), \quad p=\sigma t /(2 k), \\
& u_{1}=\operatorname{am} p, \quad u_{2}=\operatorname{am} \tau, \\
& s_{i}=\sin u_{i}, c_{i}=\cos u_{i}, d_{i}=\sqrt{1-k^{2} s_{i}^{2}}, \quad i=1,2, \\
& E_{1}=E\left(u_{1}, k\right), F_{1}=F\left(u_{1}, k\right), \Delta=1-k^{2} s_{1}^{2} s_{2}^{2} .
\end{aligned}
$$

Lemma 4.12. Suppose $\nu_{n} \in\left(D_{1} \cap N_{1}\right) \cup\left(D_{1} \cap N_{2}\right)$. If $\Delta \rightarrow 0$, then $\frac{d_{1} d_{2}}{\Delta}$ and $\frac{c_{1} d_{1}}{\Delta}$ are bounded from above.

Proof. Let $k^{2}=1-c_{3}^{2}$; then $c_{i} \rightarrow 0, \quad i=1,2,3$. Introduce spherical coordinates as follows:

$$
c_{1}=r \sin \varphi_{1} \cos \varphi_{2}, \quad c_{2}=r \sin \varphi_{1} \sin \varphi_{2}, \quad c_{3}=r \cos \varphi_{1} .
$$

Then it follows from $r \rightarrow 0$ that:

$$
\begin{aligned}
& d_{i}^{2}=1-\left(1-c_{3}^{2}\right)\left(1-c_{i}^{2}\right)=c_{i}^{2}+c_{3}^{2}-c_{i}^{2} c_{3}^{2} \approx c_{i}^{2}+c_{3}^{2}, \quad i=1,2, \\
& \Delta=1-\left(1-c_{3}^{2}\right)\left(1-c_{1}^{2}\right)\left(1-c_{2}^{2}\right) \approx c_{1}^{2}+c_{2}^{2}+c_{3}^{2}=r^{2} .
\end{aligned}
$$

This implies that

$$
\begin{aligned}
& \left(\frac{d_{1} d_{2}}{\Delta}\right)^{2} \approx \frac{\left(c_{1}^{2}+c_{3}^{2}\right)\left(c_{2}^{2}+c_{3}^{2}\right)}{r^{4}}=\left(\sin ^{2} \varphi_{1} \cos ^{2} \varphi_{2}+\cos ^{2} \varphi_{1}\right)\left(\sin ^{2} \varphi_{1} \sin ^{2} \varphi_{2}+\cos ^{2} \varphi_{1}\right) \leq 1, \\
& \left(\frac{c_{1} d_{1}}{\Delta}\right) \approx \sin ^{2} \varphi_{1} \cos ^{2} \varphi_{2}\left(\sin ^{2} \varphi_{1} \cos ^{2} \varphi_{2}+\cos ^{2} \varphi_{1}\right) \leq 1 .
\end{aligned}
$$

Therefore $\frac{d_{1} d_{2}}{\Delta}, \frac{c_{1} d_{1}}{\Delta}$ are bounded from above.

In the next lemmas we use the following parametrization of exponential mapping for the case $\lambda \in C_{1}$ (see $(2.15))$ :

$$
\begin{aligned}
x= & -\frac{4 \sigma k s_{1} s_{2} d_{1} d_{2}}{\alpha \Delta}, \\
y= & -\frac{4 \sigma}{\alpha}\left(\frac{k^{2} s_{1} s_{2}^{2} c_{1} d_{1}}{\Delta}+\frac{F_{1}}{2}-E_{1}\right), \\
z= & \frac{4 k c_{2} f_{z}}{|\alpha| \Delta}, \quad f_{z}=c_{1}\left(F_{1}-2 E_{1}\right)+s_{1} d_{1}, \\
v= & \frac{y^{3}}{6}-\frac{2 k^{2}\left(c_{1} c_{2}+s_{1} s_{2} d_{1} d_{2}\right) y}{|\alpha| \Delta^{2}}+\frac{4}{3 \alpha \sigma}\left(F_{1}\left(1-k^{2}\right)-E_{1}\left(1-2 k^{2}\right)\right. \\
& \left.-\frac{k^{2} s_{1} d_{1}}{\Delta^{3}}\left(6 s_{1} s_{2} c_{2} d_{1} d_{2}\left(2 d_{2}-\Delta\right)+c_{1}\left(1+3 c_{2}^{2}\left(d_{2}^{2}-s_{2}^{2}\right)-k^{4} s_{1}^{2} s_{2}^{6}\left(2 d_{1}^{2}+s_{1}^{2}\right)\right)\right)\right) .
\end{aligned}
$$

Lemma 4.13. If $\left\{\nu_{n}\right\} \subset D_{1} \cap N_{1}$ satisfies $\nu_{n} \rightarrow \bar{\nu} \in \operatorname{cl}\left(D_{1}\right) \backslash D_{1} \quad$ and $\quad \operatorname{Exp}\left(\nu_{n}\right) \rightarrow \bar{q} \in M_{1}$, then $\Delta \rightarrow 0$ or $\sigma \rightarrow 0$. 
Proof. Notice that for $\bar{\nu}=(\bar{x}, \bar{y}, \bar{z}, \bar{v}) \in M_{1}$ we have $\bar{x} \neq 0$ and $\bar{z} \neq 0$. Consider all possible cases $\nu_{n} \rightarrow \partial D_{1}$ :

1) $\theta_{t / 2} \rightarrow 0 \Rightarrow\left\{\begin{array}{c}\sin \frac{\theta_{t / 2}}{2} \rightarrow 0 \\ \cos \frac{\theta_{t / 2}}{2} \rightarrow 1\end{array} \Rightarrow\left\{\begin{array}{c}k s_{2} \rightarrow 0, \\ d_{2} \rightarrow 1 .\end{array}\right.\right.$ It follows that $x \approx \frac{\left(k s_{2}\right) s_{1} d_{1}}{\sigma} \Rightarrow\left[\begin{array}{r}\sigma \rightarrow 0, \\ x \rightarrow \bar{x}=0 .\end{array}\right.$

2) $\theta_{t / 2} \rightarrow \pi \Rightarrow\left\{\begin{array}{c}\sin \frac{\theta_{t / 2}}{2} \rightarrow 1 \\ \cos \frac{\theta_{t / 2}}{2} \rightarrow 0\end{array} \Rightarrow\left\{\begin{array}{r}k s_{2} \rightarrow 1, \\ d_{2} \rightarrow 0 .\end{array}\right.\right.$ Then $x \approx \frac{s_{1} d_{1} d_{2}}{\sigma \Delta} \Rightarrow\left[\begin{array}{c}\sigma \Delta \rightarrow 0 \\ x \rightarrow \bar{x}=0\end{array}\right.$

3) $c_{t / 2} \rightarrow 0 \Rightarrow k \sigma c_{2} \rightarrow 0 \Rightarrow z \approx \frac{k c_{2} f_{z}}{\sigma^{2} \Delta} \Rightarrow \frac{\sigma^{3} \Delta}{f_{z}} \rightarrow 0$, otherwise $z \rightarrow \bar{z}=0$. This means that $\sigma^{3} \Delta \rightarrow 0$ or $f_{z} \rightarrow \infty$. Suppose $f_{z} \rightarrow \infty, \sigma \rightarrow \bar{\sigma} \neq 0$, then $u_{1} \rightarrow \pi / 2$ and $k \rightarrow 1$. Since $k \sigma c_{2} \rightarrow 0$, then $u_{2} \rightarrow \pi / 2 \Rightarrow \Delta \rightarrow 0$.

4) $c_{t / 2} \rightarrow \infty \Rightarrow k \sigma c_{2} \rightarrow \infty \Rightarrow \sigma \rightarrow \infty \Rightarrow \Delta \rightarrow 0$, otherwise $x \rightarrow \bar{x}=0$.

5) $t \rightarrow 0 \Rightarrow \frac{p}{\sigma} \rightarrow 0 \Rightarrow\left[\begin{array}{l}u_{1} \rightarrow 0 \\ \sigma \rightarrow \infty\end{array} \Rightarrow \Delta \rightarrow 0\right.$, otherwise $x \rightarrow \bar{x}=0$.

6) $t \rightarrow t_{\mathrm{MAX}}^{1} \Rightarrow\left[\begin{array}{r}f_{z}\left(u_{1}, k\right) \rightarrow 0 \text { for } k \geq k_{0} \\ u_{1} \rightarrow \pi \text { for } k \leq k_{0}\end{array} \Rightarrow\left[\begin{array}{l}\sigma^{2} \Delta \rightarrow 0, \text { otherwise } z \rightarrow \bar{z}=0 \\ \sigma \Delta \rightarrow 0, \text { otherwise } x \rightarrow \bar{x}=0 .\end{array}\right.\right.$

7) $|\alpha| \rightarrow \infty \Rightarrow \sigma \rightarrow \infty \Rightarrow \Delta \rightarrow 0$, otherwise $x \rightarrow \bar{x}=0$.

Lemma 4.14. Suppose $\nu_{n} \in D_{1} \cap N_{1}$. If $\Delta \rightarrow 0$, then $x \rightarrow 0$ or $y \rightarrow \infty$.

Proof. Consider two possible cases:

1) $\sigma \rightarrow \infty \Rightarrow x=-4 k s_{1} s_{2} \frac{d_{1} d_{2}}{\Delta} \frac{1}{\sigma} \rightarrow 0$ (see Lem. 4.12).

2) $\sigma \rightarrow \bar{\sigma}<\infty$. It follows from Lemma 4.12 that $k^{2} s_{1} s_{2}^{2} \frac{c_{1} d_{1}}{\Delta}-E_{1}$ is bounded from above. Since $F_{1} \rightarrow \infty$, then $y \rightarrow \infty$.

Lemma 4.15. Suppose $\nu_{n} \in D_{1} \cap N_{1}$. If $\sigma \rightarrow 0, \Delta \rightarrow \bar{\Delta} \neq 0$, then one of the functions $x, y, z, v$ tends to $\infty$, otherwise $x$ or $z$ tends to zero.

Proof. Assume the converse. Then notice that $k s_{1} s_{2} d_{1} d_{2} \rightarrow 0$, otherwise $x \approx \frac{1}{\sigma} \rightarrow \infty$. The proof consists of the following six items:

1) $\quad d_{1} \rightarrow 0$. This means that $u_{1} \rightarrow \pi / 2, k \rightarrow 1$ and $u_{2} \rightarrow \overline{u_{2}} \neq \pi / 2$. Whence, $y \approx F_{1} / \sigma \rightarrow \infty$.

2) $u_{1} \rightarrow 0$. Consider four subcases:

2.1) $s_{2} k \rightarrow 0$. Here we have

$$
\begin{aligned}
& x \approx \frac{s_{2} k u_{1}}{\sigma} \Rightarrow s_{2} k u_{1} \approx \sigma \\
& F_{1} \sim u_{1}, E_{1} \sim u_{1} \Rightarrow y \approx \frac{1}{\sigma}\left(s_{1}\left(s_{2} k\right)^{2} \frac{c_{1} d_{1}}{\Delta}+\frac{F_{1}}{2}-E_{1}\right) \approx \frac{u_{1}}{\sigma} \approx \frac{1}{s_{2} k} \rightarrow \infty .
\end{aligned}
$$

2.2) $d_{2} \rightarrow 0$. It follows that

$$
x \approx \frac{d_{2} u_{1}}{\sigma} \Rightarrow d_{2} u_{1} \approx \sigma, \quad y \approx \frac{u_{1}}{\sigma} \approx \frac{1}{d_{2}} \rightarrow \infty .
$$

2.3) $c_{2} \rightarrow 0, k \rightarrow \bar{k} \in(0,1)$. We get

$$
x \approx \frac{u_{1}}{\sigma} \Rightarrow u_{1} \approx \sigma, \quad z \approx \frac{c_{2} u_{1}^{3}}{\sigma^{2}} \approx c_{2} u_{1} \rightarrow 0 .
$$


2.4) $k \rightarrow \bar{k} \neq 0, u_{2} \rightarrow \overline{u_{2}} \in(0, \pi / 2)$. Hence

$$
x \approx \frac{u_{1}}{\sigma}, \quad z \approx \frac{u_{1}^{3}}{\sigma^{2}} \approx x^{2} u_{1} \rightarrow 0 .
$$

3) $u_{1} \rightarrow \pi \Rightarrow F_{1} \rightarrow 2 K, E_{1} \rightarrow 2 E$. We obtain $y \approx \frac{2 E-K}{\sigma} \Rightarrow 2 E-K \rightarrow 0$. It follows that $v \approx \frac{K}{\sigma^{3}} \rightarrow \infty$, since $K \rightarrow \bar{K}=K(\bar{k})>0$.

4) $d_{2} \rightarrow 0, u_{1} \rightarrow \overline{u_{1}} \in(0, \pi / 2) \Rightarrow u_{2} \rightarrow \pi / 2, k \rightarrow 1$.

$$
x \approx \frac{d_{2}}{\sigma}, \quad y \approx \frac{1}{\sigma}\left(s_{1}+\frac{F_{1}}{2}-E_{1}\right) .
$$

Note that the function $\sin u_{1}+\frac{F\left(u_{1}, 1\right)}{2}-E\left(u_{1}, 1\right)$ vanishes only at the point $u_{1}=0$ since it has positive derivative $\frac{1}{2 \sqrt{1-\sin ^{2} u_{1}}}$. Therefore $y \rightarrow \infty$.

5) $\quad u_{2} \rightarrow 0, s_{1} \rightarrow \overline{s_{1}} \neq 0, d_{1} \rightarrow \overline{d_{1}} \neq 0, k \rightarrow \bar{k} \neq 0$. We have $y \approx \frac{2 E_{1}-F_{1}}{\sigma} \Rightarrow 2 E_{1}-F_{1} \rightarrow 0$. Hence $z \approx \frac{1}{\sigma^{2}} \rightarrow \infty$.

6) $\quad k \rightarrow 0$. We get $y \approx \frac{u_{1}}{\sigma} \Rightarrow u_{1} \rightarrow 0$ (see item 2.1).

In the next lemmas we use the following parametrization of exponential mapping for the case $\lambda \in C_{2}$ (see $(2.16))$ :

$$
\begin{aligned}
x= & -\frac{4 \operatorname{sgn} c \sigma s_{1} s_{2} c_{1} c_{2}}{\alpha k \Delta}, \\
y= & -\frac{4 \sigma}{\alpha k}\left(\frac{k^{2} s_{1} s_{2}^{2} c_{1} d_{1}}{\Delta}+\left(1-\frac{k^{2}}{2}\right) F_{1}-E_{1}\right) \\
z= & -4 \frac{4 \operatorname{sgn} c d_{2} g_{z}}{|\alpha| k^{2} \Delta}, \quad g_{z}=\left(2 E_{1}+\left(k^{2}-2\right) F_{1}\right) d_{1}-k^{2} s_{1} c_{1}, \\
v= & \frac{y^{3}}{6}+\frac{2 y}{|\alpha| k^{2} \Delta^{2}}\left(1+\left(1+c_{1}^{2} c_{2}^{2}\right) k^{4} s_{1}^{2} s_{2}^{2}-k^{2}\left(s_{1}^{2}+s_{2}^{2}-2 c_{1} c_{2} d_{1} d_{2} s_{1} s_{2}\right)\right) \\
& -\frac{3}{4 \alpha \sigma k}\left(2 F_{1}\left(\frac{1}{k^{2}}-1\right)-E_{1}\left(\frac{2}{k^{2}}-1\right)+\frac{c_{1} s_{1}}{\Delta^{3}}\left(2 c_{2}^{2} d_{1}\left(1+d_{1}^{2}\right) \Delta+6 c_{1} c_{2} d_{2} k^{2} s_{1} s_{2}\left(2 c_{2}^{2}-\Delta\right)\right.\right. \\
& \left.\left.+d_{1} s_{2}^{2}\left(\left(2-k^{2}\right) \Delta^{2}-4 d_{1}^{2} d_{2}^{2}\right)\right)\right) .
\end{aligned}
$$

Lemma 4.16. If $\left\{\nu_{n}\right\} \subset D_{1} \cap N_{2}$ satisfies $\nu_{n} \rightarrow \bar{\nu} \in \operatorname{cl}\left(D_{1}\right) \backslash D_{1}$ and $\operatorname{Exp}\left(\nu_{n}\right) \rightarrow \bar{q} \in M_{1}$, then $\Delta \rightarrow 0$ or $\frac{\sigma}{k} \rightarrow 0$.

Proof. Notice that for $\bar{\nu}=(\bar{x}, \bar{y}, \bar{z}, \bar{v}) \in M_{1}$ we have $\bar{x} \neq 0$. Consider all possible cases for $\bar{\nu} \in \operatorname{cl}\left(D_{1}\right) \backslash D_{1}$ :

1) $\theta_{t / 2} \rightarrow 0 \Rightarrow\left\{\begin{array}{l}\sin \frac{\theta_{t / 2}}{2} \rightarrow 0 \\ \cos \frac{\theta_{t / 2}}{2} \rightarrow 1\end{array} \Rightarrow\left\{\begin{array}{l}s_{u_{2}} \rightarrow 0, \\ c_{u_{2}} \rightarrow 1 .\end{array}\right.\right.$ It follows that $\frac{\sigma \Delta}{k} \rightarrow 0$, otherwise $x \rightarrow \bar{x}=0$.

2) $\theta_{t / 2} \rightarrow \pi \Rightarrow\left\{\begin{array}{l}\sin \frac{\theta_{t / 2}}{2} \rightarrow 1 \\ \cos \frac{\theta_{t / 2}}{2} \rightarrow 0\end{array} \Rightarrow\left\{\begin{array}{l}s_{2} \rightarrow 1, \\ c_{2} \rightarrow 0 .\end{array}\right.\right.$ This means that $\frac{\sigma \Delta}{k} \rightarrow 0$, otherwise $x \rightarrow \bar{x}=0$.

3) $c_{t / 2} \rightarrow 0 \Rightarrow \frac{\sigma}{k} d_{2} \rightarrow 0 \Rightarrow \frac{\sigma}{k} \rightarrow 0$ or $c_{2} \rightarrow 0$. From $c_{2} \rightarrow 0$ we have $\frac{\sigma \Delta}{k} \rightarrow 0$, otherwise $x \rightarrow \bar{x}=0$. 
4) $c_{t / 2} \rightarrow \infty \Rightarrow \frac{\sigma}{k} d_{2} \rightarrow \infty \Rightarrow \frac{\sigma}{k} \rightarrow \infty \Rightarrow \Delta \rightarrow 0$, otherwise $x \rightarrow \bar{x}=0$.

5) $t \rightarrow 0 \Rightarrow \frac{p k}{\sigma} \rightarrow 0 \Rightarrow \frac{s_{1} k}{\sigma} \rightarrow 0 \Rightarrow \Delta \rightarrow 0$, otherwise $x \rightarrow \bar{x}=0$.

6) $t \rightarrow t_{\mathrm{MAX}}^{1} \Rightarrow u_{1} \rightarrow \frac{\pi}{2} \Rightarrow \frac{\sigma \Delta}{k} \rightarrow 0$, otherwise $x \rightarrow \bar{x}=0$.

7) $\alpha \rightarrow \infty \Rightarrow \sigma \rightarrow \infty \Rightarrow \Delta \rightarrow 0$, otherwise $x \rightarrow \bar{x}=0$.

Lemma 4.17. Suppose $\nu_{n} \in D_{1} \cap N_{2}$. If $\Delta \rightarrow 0$, then $x \rightarrow 0$ or $y \rightarrow \infty$.

Proof. Consider two possible cases:

1) $\sigma \rightarrow \infty \Rightarrow x=-4 k s_{1} s_{2} \frac{c_{1} c_{2}}{\Delta} \frac{1}{\sigma} \rightarrow 0$ (see Lem. 4.12).

2) $\sigma \rightarrow \bar{\sigma}<\infty$. It follows from Lemma 4.12 that $\left(k^{2} s_{1} s_{2}^{2} \frac{c_{1} d_{1}}{\Delta}-E_{1}\right)$ is bounded from above. And since $F_{1} \rightarrow \infty$ we have $y \rightarrow \infty$.

Lemma 4.18. Suppose $\nu_{n} \in D_{1} \cap N_{2}$. If $\frac{\sigma}{k} \rightarrow 0, \Delta \rightarrow \bar{\Delta} \neq 0$, then one of the functions $x, y, z$ or $v$ tends to $\infty$, otherwise $x$ or $z$ tends to 0 .

Proof. Assume the converse. Then notice that $s_{1} s_{2} c_{1} c_{2} \rightarrow 0$, otherwise $x \approx \frac{k}{\sigma} \rightarrow \infty$. The proof consists of five steps:

1) $u_{1} \rightarrow 0$. Then we obtain

$$
x \approx \frac{u_{1} s_{1} c_{2}}{\sigma / k} \Rightarrow \frac{\sigma}{k} \approx u_{1} s_{2} c_{2}
$$

It follows from Taylor expansion that $g_{z} \approx k^{2} u_{1}^{3}$, then

$$
z \approx \frac{d_{2} k^{2} u_{1}^{3}}{\sigma^{2}} \approx \frac{d_{2} u_{1}}{s_{2}^{2} c_{2}^{2}}=\frac{d_{2} u_{1}}{s_{2}^{2} c_{2}^{2}} \Rightarrow s_{2} c_{2} \rightarrow 0
$$

otherwise $z \rightarrow 0$.

$$
y \approx \frac{1}{\sigma k}\left(k^{2} u_{1} s_{2}^{2}+\left(1-k^{2} / 2\right) u_{1}-u_{1}\right)=\frac{u_{1}\left(s_{2}^{2}-1 / 2\right)}{\sigma / k} \approx \frac{s_{2}^{2}-1 / 2}{s_{2} c_{2}} \rightarrow \infty .
$$

2) $k \rightarrow 0, u_{1} \rightarrow \overline{u_{1}} \neq 0$. Using Taylor expansion we get $z \approx \frac{k^{2}}{\sigma^{2}} \rightarrow \infty$.

3) $u_{1} \rightarrow \frac{\pi}{2}, k \rightarrow \bar{k} \neq 0$. We have $y \approx \frac{1}{\sigma}\left(\frac{k^{2} c_{1} s_{1} d_{1}^{2} s_{2}^{2}}{\Delta}+\left(1-k^{2} / 2\right) F_{1}-E_{1}\right)$. Notice that

$$
\frac{\mathrm{d}}{\mathrm{d} k}\left(\left(1-k^{2} / 2\right) F_{1}-E_{1}\right)=\frac{k}{2\left(1-k^{2}\right)} \int_{0}^{\pi / 2} \frac{k^{2} \cos ^{2} \theta \mathrm{d} \theta}{\sqrt{1-k^{2} \sin ^{2} \theta}}>0 \Rightarrow\left(1-k^{2} / 2\right) F_{1}-E_{1}>0 .
$$

Combining the last inequality and $\frac{k^{2} c_{1} s_{1} d_{1}^{2} s_{2}^{2}}{\Delta} \rightarrow 0$, we obtain $y \rightarrow \infty$.

4) $u_{2} \rightarrow 0, u_{1} \rightarrow \overline{u_{1}} \in(0, \pi / 2), k \rightarrow \bar{k} \neq 0$. Here we have

$$
x \approx \frac{u_{2}}{\sigma} \Rightarrow u_{2} \approx \sigma
$$


otherwise $x \rightarrow 0$.

$$
\begin{aligned}
& z \approx \frac{\left(2 E_{1}+\left(k^{2}-2\right) F_{1}\right) d_{1}-k^{2} c_{1} s_{1}}{\sigma^{2}}, \\
& y \approx \frac{1}{\sigma}\left(k^{2} s_{1} c_{1} d_{1} s_{2}^{2}+\left(1-k^{2} / 2\right) F_{1}-E_{1}\right) .
\end{aligned}
$$

Since $k^{2} s_{1} c_{1} d_{1} s_{2}^{2} \rightarrow 0$, we see that $\left(1-k^{2} / 2\right) F_{1}-E_{1} \rightarrow 0$, otherwise $y \rightarrow \infty$. Hence from $k^{2} c_{1} s_{1} \approx 1$ we get $z \approx \frac{1}{\sigma^{2}} \rightarrow \infty$.

5) $u_{2} \rightarrow \pi / 2, u_{1} \rightarrow u_{1} \in(0, \pi / 2), k \rightarrow \bar{k} \neq 0$. Suppose $\bar{k} \neq 1$, then $z \approx \frac{d_{2} g_{z}}{\sigma^{2}} \rightarrow \infty$. This means that $\bar{k}=1$. Here we have $y \approx \frac{1}{\sigma}\left(\frac{c_{1} s_{1} d_{1}}{\Delta}+F_{1} / 2-E_{1}\right)=\frac{1}{\sigma}\left(s_{1}+F_{1} / 2-E_{1}\right)$. Since $\frac{\mathrm{d}}{\mathrm{d} u_{1}}\left(s_{1}+F_{1} / 2-E_{1}\right)=\frac{1}{2 \cos u_{1}}>0$, it follows that $y \rightarrow \infty$.

Theorem 4.19. The mapping $\operatorname{Exp}: D_{i} \rightarrow M_{i}$ is proper for $i=1, \ldots, 4$.

Proof. Assume the converse. Then it follows from Lemma 4.1 that Exp : $D_{1} \rightarrow M_{1}$ is not proper. By Lemma 4.2, there exists a sequence $\nu_{n} \in D_{1}$ such that $\nu_{n} \rightarrow \bar{\nu} \in \operatorname{cl}\left(D_{1}\right) \backslash D_{1}$, $\operatorname{Exp}\left(\nu_{n}\right) \rightarrow \bar{q} \in M_{1}$. Since $\nu_{n} \in D_{1}$, we consider 3 cases:

1) $\left\{\nu_{n}\right\} \subset D_{1} \cap N_{6}$ is impossible (see Lems. 4.7, 4.8),

2) $\left\{\nu_{n}\right\} \subset D_{1} \cap N_{3}$ is impossible (see Lems. 4.9-4.11),

3) $\left\{\nu_{n}\right\} \subset\left(D_{1} \cap N_{1}\right) \cup\left(D_{1} \cap N_{2}\right)$ is impossible (see Lems. 4.13-4.18).

Since all cases are impossible, we have a contradiction which proves the theorem.

Theorem 4.20. The mapping Exp $: D_{i} \rightarrow M_{i}$ is a diffeomorphism for $i=1, \ldots, 4$.

Proof. Follows from Theorem 3.5, since all hypotheses of this theorem hold by Proposition 3.7, Theorems 2.3 and 4.19 .

Corollary 4.21. The mapping $\operatorname{Exp}: \widetilde{N} \rightarrow \widetilde{M}$ is a diffeomorphism.

\section{Cut time}

In this section we prove that the cut time coincides with the first Maxwell time corresponding to reflections.

\subsection{Cut time and Maxwell time}

Theorem 5.1. For any $\lambda \in C$,

$$
t_{\text {cut }}(\lambda)=t_{\text {MAX }}^{1}(\lambda)
$$

Proof. Take any $\lambda \in C$ and denote $t_{1}=t_{\mathrm{MAX}}^{1}(\lambda)$. Since $t_{\text {cut }}(\lambda) \leq t_{1}$ by Theorem 2.1 , it remains to prove that $t_{\text {cut }}(\lambda) \geq t_{1}$.

Let us call a pair $(\lambda, t) \in N$ optimal if the geodesic $\operatorname{Exp}(\lambda, s)$ is optimal on the segment $s \in[0, t]$. We have to show that $(\lambda, t)$ is optimal for any $t \in\left(0, t_{1}\right)$.

1) If $\lambda \in C_{4} \cup C_{5} \cup C_{7}$, then $t_{1}=+\infty$, and any $(\lambda, t), t \in\left(0, t_{1}\right)$, is optimal since $\left(x_{s}, y_{s}\right)$ is a straight line.

2) Let $\lambda \in C_{1} \cup C_{2} \cup C_{6}$, thus $t_{1} \in(0,+\infty)$.

Since $t_{1}=t_{\mathrm{MAX}}^{1}(\lambda)$, then $\nu_{1}=\left(\lambda, t_{1}\right) \in N^{\prime}$. For $\lambda \in C_{1} \cup C_{2} \cup C_{6}$ the function $t \mapsto \sin \theta_{t / 2} c_{t / 2}$ has isolated zeros, thus there exists $t \in\left(0, t_{1}\right)$ arbitrarily close to $t_{1}$ such that $\nu=(\lambda, t) \in \widetilde{N}$. Then $q=\operatorname{Exp}(\nu) \in \widetilde{M}$ (Prop. 3.4). Since $\operatorname{Exp}\left(N^{\prime}\right) \cap \widetilde{M}=\emptyset$ (Prop. 3.3) and $\operatorname{Exp}: \widetilde{N} \rightarrow \widetilde{M}$ is a diffeomorphism (see Cor. 4.21) then $\operatorname{Exp}^{-1}(q) \cap \widehat{N}=\{\nu\}$. Thus $\nu=(\lambda, t)$ is optimal. Since $t$ can be chosen arbitrarily close to $t_{1}$, then any $(\lambda, t), t \in\left(0, t_{1}\right)$, is optimal. 
3) Let $\lambda \in C_{3}$, then $t_{1}=+\infty$. There exist $(\lambda, t) \in \widetilde{N}$ for arbitrarily large $t$. Then the proof follows the argument of item 2).

Now we collect all properties of the cut time that we previously obtained for the Maxwell time $t_{\mathrm{MAX}}^{1}$.

Corollary 5.2. The function $t_{\mathrm{cut}}: C \rightarrow(0,+\infty]$ has the following properties:

1) Let $\lambda \in C$ and let $t_{1}=t_{\text {cut }}(\lambda)$. For finite $t_{1}$, a trajectory $\operatorname{Exp}(\lambda, s), s \in[0, t]$, is optimal iff $t \in\left[0, t_{1}\right]$. For $t_{1}=+\infty$, any trajectory $\operatorname{Exp}(\lambda, s), s \in[0, t], t>0$, is optimal.

2) The function $t_{\text {cut }}$ has the following explicit representation:

$$
\begin{array}{ll}
\forall \lambda \in C_{1} & t_{\text {cut }}(\lambda)=\frac{\min \left(2 p_{z}^{1}, 4 K\right)}{\sqrt{|\alpha|}}, \\
\forall \lambda \in C_{2} & t_{\text {cut }}(\lambda)=\frac{2 K k}{\sqrt{|\alpha|},} \\
\forall \lambda \in C_{6} & t_{\text {cut }}(\lambda)=\frac{2 \pi}{|c|}, \\
\forall \lambda \in C_{3} \cup C_{4} \cup C_{5} \cup C_{7} & t_{\text {cut }}(\lambda)=+\infty .
\end{array}
$$

3) The function $t_{\mathrm{cut}}$ depends only on $E$ and $|\alpha|$, is preserved by the flow of $\overrightarrow{\mathrm{H}}_{v}$ and by the reflections $\varepsilon^{i}$, and is homogeneous of order one w.r.t. the dilations $\delta_{\mu}$.

4) The function $t_{\text {cut }}$ is continuous on $C \backslash C_{4}$ and is smooth on $C_{1}^{0} \cup C_{2}$, where $C_{1}^{0}=\left\{\lambda \in C_{1} \mid k \neq k_{0}\right\}$.

According to Corollary 5.2, the function $t_{\text {cut }}: C \rightarrow(0,+\infty]$ is invariant w.r.t. the flow $e^{s \overrightarrow{\mathrm{H}}_{v}}$ and the reflections $\varepsilon^{i}$, and respects the action of dilations $\delta_{\mu}$ :

$$
\begin{aligned}
& t_{\text {cut }} \circ \mathrm{e}^{s \overrightarrow{\mathrm{H}}_{v}}=t_{\text {cut }} \circ \varepsilon^{i}=t_{\text {cut }}, \\
& t_{\text {cut }} \circ \delta_{\mu}=\mu t_{\text {cut }} .
\end{aligned}
$$

Thus the cut time can be represented (up to a constant positive factor) by a univariate function on the quotient $C /\left\langle\mathrm{e}^{s \overrightarrow{\mathrm{H}}_{v}}, \varepsilon^{i}, \delta_{\mu}\right\rangle$. The quotient $C /\left\langle\mathrm{e}^{s \overrightarrow{\mathrm{H}}_{v}}, \varepsilon^{i}\right\rangle$ can be represented by the quadrant $\{(\theta, c, \alpha) \in C \mid \theta=0, c \geq$ $0, \alpha \geq 0\}$, thus

$$
C /\left\langle\mathrm{e}^{s \overrightarrow{\mathrm{H}_{v}}}, \varepsilon^{i}, \delta_{\mu}\right\rangle \cong \Gamma \sqcup P
$$

where

$$
\begin{aligned}
& \Gamma=\{(\theta, c, \alpha) \in C \mid \theta=0, c=\sin \beta, \alpha=\cos \beta, \beta \in[0, \pi / 2]\}, \\
& P=\{(\theta, c, \alpha) \in C \mid \theta=0, c=0, \alpha=0\} .
\end{aligned}
$$

The point $P$ corresponds to the subset $C_{7}$, while the arc $\Gamma$ corresponds to the rest subsets $C \backslash C_{7}=C_{1} \cup C_{2} \cup$ $C_{35} \cup C_{4} \cup C_{6}$ of decomposition (2.36).

Thus (up to a constant positive factor) the cut time can be represented on the set $C \backslash C_{7}$ as a univariate function $t_{\text {cut }}(\beta), \beta \in[0, \pi / 2]$.

If $\beta=0$, then $\lambda \in C_{4}$, thus $t_{\text {cut }}(\beta)=+\infty$.

If $\beta \in\left(0, \beta_{1}\right)$, where $\beta_{1}=\arccos (\sqrt{5}-2)$, then $\lambda \in C_{1}$, thus

$$
t_{\text {cut }}(\beta)=\frac{2 p_{1}(k)}{\sqrt{\alpha}}, \quad k=\sqrt{\frac{\sin ^{2} \beta}{4 \cos \beta}}, \quad \alpha=\cos \beta, \quad p_{1}(k)=\min \left(p_{z}^{1}(k), 2 K(k)\right) .
$$

If $\beta=\beta_{1}$, i.e., $\sin ^{2} \beta=4 \cos \beta$, then $\lambda \in C_{35}$, thus $t_{\text {cut }}(\beta)=+\infty$. 


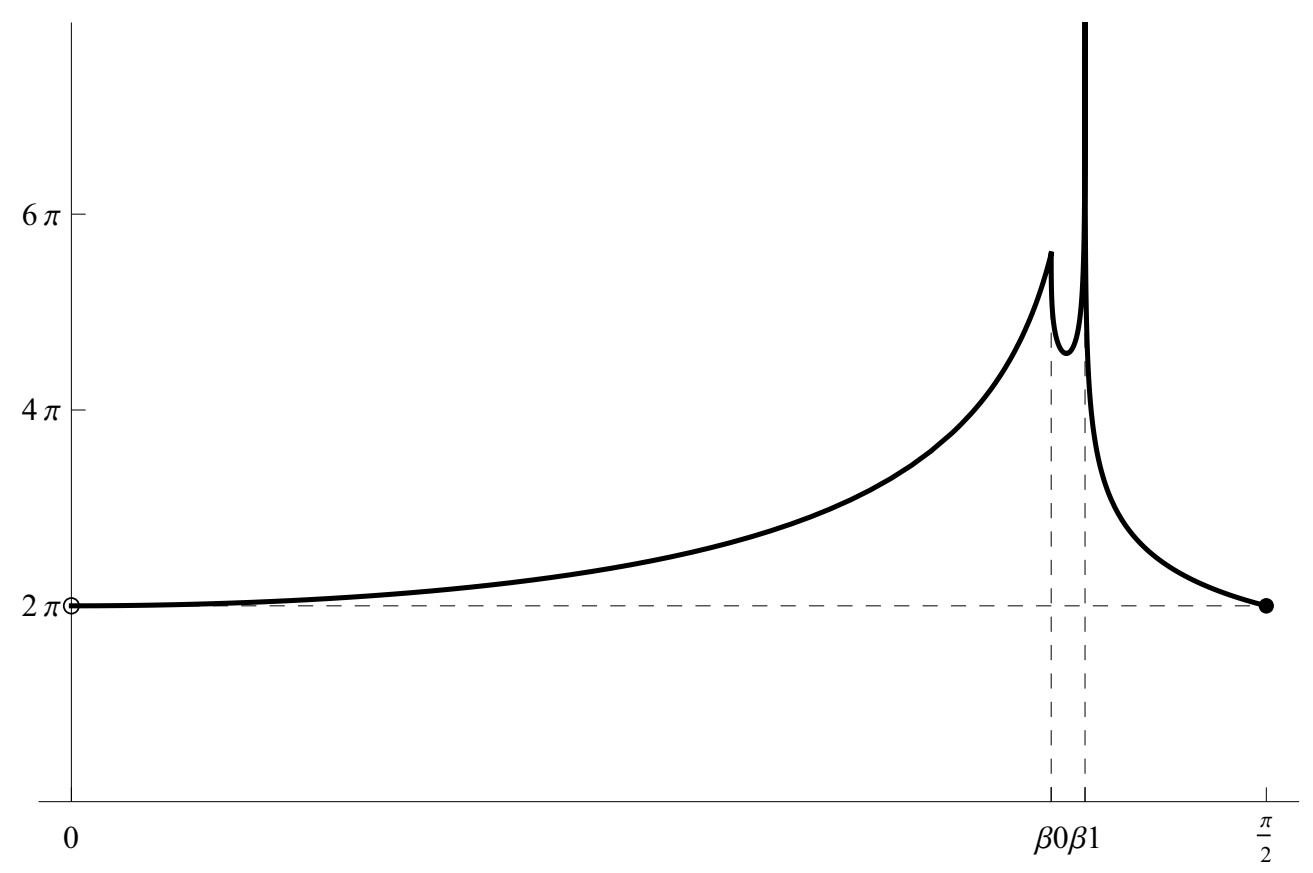

Figure 3. Plot of the function $\beta \mapsto t_{\text {cut }}(\beta)$.

If $\beta \in\left(\beta_{1}, \pi / 2\right)$, then $\lambda \in C_{2}$, thus

$$
t_{\text {cut }}(\beta)=\frac{2 K k}{\sqrt{\alpha}}, \quad k=\sqrt{\frac{4 \cos \beta}{\sin ^{2} \beta}}, \quad \alpha=\cos \beta .
$$

Finally, if $\beta=\pi / 2$, then $\lambda \in C_{6}$, thus $t_{\text {cut }}(\beta)=2 \pi$.

The plot of the function $t_{\text {cut }}(\beta)$ is shown in Figure 3. Notice continuity of $t_{\text {cut }}(\beta)$ everywhere except $\beta=0$, where $t_{\text {cut }}(+0)=2 \pi<+\infty=t_{\text {cut }}(0)$. Also notice smoothness of $t_{\text {cut }}(\beta)$ everywhere except $\beta=0, \beta=\beta_{1}$, and $\beta=\beta_{0}$, where $\frac{\sin ^{2} \beta_{0}}{4 \cos \beta_{0}}=k_{0}^{2}, 2 E\left(k_{0}\right)-K\left(k_{0}\right)=0$ (here $E\left(k_{0}\right)$ is the complete elliptic integral of the second kind), corresponds to the figure-of-eight closed Euler elastica. These regularity properties of $t_{\text {cut }}(\lambda)=t_{\text {MAX }}^{1}(\lambda)$ are reported in Corollary 5.2.

\subsection{Cut time and conjugate time}

Proposition 5.3. Let $\lambda \in C_{1}, t_{1}=t_{\mathrm{cut}}(\lambda), \tau=\left(\varphi+t_{1} / 2\right) / \sqrt{|\alpha|}$. Then $t_{1}=t_{\text {conj }}^{1}(\lambda)$ iff one of the conditions hold:

1) $k<k_{0}$, sn $\tau=0$,

2) $k=k_{0}$,

3) $k>k_{0}$, cn $\tau=0$.

In particular, if $t_{1}=t_{\text {conj }}^{1}(\lambda)$, then $\operatorname{sn} \tau \operatorname{cn} \tau=0$ or $k=k_{0}$.

Proof. Follows immediately from Lemma 8 of [7].

Remark 5.4. The equality $\operatorname{sn} \tau=0(\operatorname{cn} \tau=0)$ is equivalent to $\sin \theta_{t / 2}=0\left(c_{t_{1} / 2}=0\right)$; it means that elastica $\left(x_{t}, y_{t}\right), t \in\left[0, t_{1}\right]$, is centered at a vertex (resp. inflexion point). The equality $k=k_{0}$ means that $\left(x_{t}, y_{t}\right), t \in$ $\left[0, t_{1}\right]$, is the closed figure-of-eight elastica. 
Proposition 5.5. Let $\lambda \in C_{2}, t_{1}=t_{\text {cut }}(\lambda), \tau=\left(\varphi+t_{1} / 2\right) /(k \sqrt{|\alpha|})$. Then $t_{1}=t_{\text {conj }}^{1}(\lambda)$ iff $\operatorname{sn} \tau \operatorname{cn} \tau=0$.

Proof. Follows immediately from Lemma 8 of [7].

Remark 5.6. The equality $\operatorname{sn} \tau \operatorname{cn} \tau=0$ is equivalent to $\sin \theta_{t / 2}=0$. It means that the corresponding elastica is centered at vertex.

Proposition 5.7. Let $\lambda \in C_{6}, \quad t_{1}=t_{\text {cut }}(\lambda)$. Then $t_{1}=t_{\text {conj }}^{1}(\lambda)$ iff $\sin \theta=0$.

Proof. Let $\lambda=(\theta, c, \alpha) \in C_{6}, \alpha=0, c \neq 0, t_{1}=t_{\text {cut }}(\lambda), \nu=\left(\lambda, t_{1}\right) \in N_{6}$. Since $C_{6} \subset \operatorname{cl}\left(C_{2}\right)$, the expression for Jacobian $J=\frac{\partial(x, y, z, v)}{\partial(\theta, c, \alpha, t)}$ for $\nu \in N_{6}$ can be obtained by passing to the limit $\alpha \rightarrow 0$ in the expression for Jacobian $\left.J\right|_{N_{2}}$ computed in [7]. By such a limit we get $J(\nu)=\frac{\pi^{3}}{|c|^{3}} \sin ^{2} \theta$. So the instant $t_{1}$ is a conjugate time iff $\sin \theta=0$.

\subsection{Optimal trajectories for special boundary conditions}

For a generic terminal point $q_{1} \in \widetilde{M}$, there exists a unique optimal trajectory $q_{t}=\operatorname{Exp}(\lambda, t), t \in\left[0, t_{1}\right]$, which can be found by solving the equation $\operatorname{Exp}\left(\lambda, t_{1}\right)=q_{1},\left(\lambda, t_{1}\right) \in \widetilde{N}$.

In this subsection we discuss special boundary conditions for which optimal trajectories can be given explicitly or by a more simple equation.

\subsubsection{Abnormal variety}

Consider the set of points in $M$ filled by abnormal trajectories:

$$
A=\left\{q \in M \mid x=z=0, v=y^{3} / 6\right\} .
$$

We have $\operatorname{Exp}\left(C_{4}, \mathbb{R}_{+}\right)=\operatorname{Exp}\left(C_{5}, \mathbb{R}_{+}\right)=\operatorname{Exp}\left(C_{7}^{0, \pi}, \mathbb{R}_{+}\right)=A \backslash\left\{q_{0}\right\}$, where

$$
C_{7}^{0, \pi}=\left\{\lambda=(\theta, c, \alpha) \in C_{7} \mid \alpha=c=0, \theta \in\{0, \pi\}\right\} .
$$

Any nonzero point $q_{1}=\left(0, y_{1}, 0, v_{1}\right) \in A$ is connected with $q_{0}$ by a unique optimal trajectory

$$
x_{t}=0, \quad y_{t}=t \operatorname{sgn} y_{1}, \quad z_{t}=0, \quad v_{t}=\frac{t^{3}}{6} \operatorname{sgn} y_{1}, \quad t \in\left[0,\left|y_{1}\right|\right] .
$$

\subsubsection{Straight lines $\left(x_{t}, y_{t}\right)$}

The set of points in $M$ filled by trajectories that project to straight lines $\left(x_{t}, y_{t}\right)$ is

$$
L=\left\{q \in M \mid z=0, v=\left(x^{2}+y^{2}\right) y / 6\right\} \supset A .
$$

We have $\operatorname{Exp}\left(C_{7}, \mathbb{R}_{+}\right)=L \backslash\left\{q_{0}\right\}$. The unique optimal trajectory for $q_{1} \in L \backslash\left\{q_{0}\right\}$ is $\operatorname{Exp}(\lambda, t), \lambda \in C_{7}$, i.e.,

$$
x_{t}=-t \sin \theta, \quad y_{t}=t \cos \theta, \quad z_{t}=0, \quad v_{t}=\frac{t^{3}}{6} \cos \theta, \quad t \in\left[0, t_{1}\right],
$$

where $t_{1}>0$ and $\theta \in S^{1}$ are found from the equations

$$
x_{1}=-t_{1} \sin \theta, \quad y_{1}=t_{1} \cos \theta .
$$




\subsubsection{Fixed points of reflection $\varepsilon^{6}$}

The reflection $\varepsilon^{6}: M \rightarrow M$ has the set of fixed points

$$
S_{6}=\{q \in M \mid y=0, v=x z / 2\} .
$$

This 2-dimensional manifold is of particular interest since it is the only fixed manifold of reflections

$$
S_{i}=\left\{q \in M \mid \varepsilon^{i}(q)=q\right\}, \quad i=1, \ldots, 7,
$$

not contained completely in codimension one manifolds $S_{1}=\{q \in M \mid z=0\}$ and $S_{2}=\{q \in M \mid x=0\}$. By Lemma 4 of [6],

$$
S_{3} \cup S_{4} \cup S_{5} \subset S_{2}, \quad S_{7} \subset S_{1} .
$$

But $S_{6} \not \subset S_{1} \cup S_{2}$.

If a point $q_{1} \in S_{6} \backslash\left\{q_{0}\right\}$ is connected with $q_{0}$ by a trajectory $q_{t}=\operatorname{Exp}(\lambda, t), t \in\left[0, t_{1}\right], q_{t_{1}}=q_{1}$, then the trajectory $q_{t}^{6}=\operatorname{Exp}\left(\lambda^{6}, t\right), t \in\left[0, t_{1}\right]$, satisfies the equation $q_{t_{1}}^{6}=q_{1}$.

Moreover, if $\lambda^{6} \neq \lambda$, then the points $\left(\lambda, t_{1}\right),\left(\lambda^{6}, t_{1}\right)$ would belong to the Maxwell set

$$
\operatorname{MAX}^{6}=\left\{(\lambda, t) \in N \mid \lambda^{i} \neq \lambda, \operatorname{Exp}\left(\lambda^{i}, t\right)=\operatorname{Exp}(\lambda, t)\right\}
$$

which might a priori give Maxwell times which are additional to those provided by the sets $\mathrm{MAX}^{1}, \mathrm{MAX}^{2}$ studied in [6]. It turns out that, as we show below, the equality $\lambda^{6}=\lambda$ is satisfied for all $\lambda \in C$ with $\operatorname{Exp}\left(\lambda, t_{1}\right) \in$ $S_{6}, t_{1}>0$.

Consider the decomposition

$$
\begin{aligned}
& S_{6}=\bigsqcup_{i, j \in\{0,+,-\}} S_{i j}, \\
& S_{i j}=\left\{q \in S_{6} \mid \operatorname{sgn} x=i, \operatorname{sgn} z=j\right\} .
\end{aligned}
$$

For example, $S_{+-}=\left\{q \in S_{6} \mid x>0, z<0\right\}$.

Denote $N_{i j}=\left\{(\lambda, t) \in N_{6} \mid \tau=-i \pi / 2, \operatorname{sgn} c=j, t \in(0,2 \pi /|c|)\right\}, \quad i, j \in\{+,-\}$, where $\tau=\theta+c t / 2$.

Lemma 5.8. For any $i, j \in\{+,-\}$, the mapping $\operatorname{Exp}: N_{i j} \rightarrow S_{i j}$ is a diffeomorphism.

Proof. The reflections $\varepsilon^{4}$ and $\varepsilon^{7}$ permute the sets $N_{i j}, S_{i j}$, thus it suffices to prove only that the mapping Exp: $N_{++} \rightarrow S_{++}$is a diffeomorphism.

If $(\lambda, t) \in N_{++}$, then

$$
x_{t}=\frac{2 \sin p}{c}, \quad y_{t}=0, \quad z_{t}=\frac{2 p-\sin (2 p)}{2 c^{2}}, \quad v_{t}=\frac{x_{t} z_{t}}{2},
$$

where $p=c t / 2 \in(0, \pi)$. Thus $\operatorname{Exp}\left(N_{++}\right) \subset S_{++}$.

Further, the mapping $\Phi:(p, c) \mapsto(x, z)$ is a diffeomorphism from $(0, \pi) \times \mathbb{R}_{+}$to $\mathbb{R}_{+} \times \mathbb{R}_{+}$by Hadamard global diffeomorphism theorem, thus Exp: $N_{++} \rightarrow S_{++}$is a diffeomorphism as well.

Denote $N^{i}{ }_{0 j}=\left\{(\lambda, t) \in N_{6}|\tau=i \pi / 2, \operatorname{sgn} c=j, t=2 \pi /| c \mid\right\}, \quad i, j \in\{+,-\}$.

Lemma 5.9. Each of the mappings Exp: $N_{0 j}^{+} \rightarrow S_{0 j}$, Exp: $N_{0 j}^{-} \rightarrow S_{0 j}, j \in\{+,-\}$, is a diffeomorphism.

Proof. Follows from the parameterization of trajectories (5.1) with $p= \pm \pi$.

Denote $N_{i 0}=\left\{(\lambda, t) \in N_{7} \mid \theta=-i \pi / 2\right\}, \quad i \in\{+,-\}$.

Lemma 5.10. The mappings $\operatorname{Exp}: N_{i 0} \rightarrow S_{i 0}, i \in\{+,-\}$, are diffeomorphisms. 
Proof. Follows immediately from the parameterization of extremal trajectories for $\lambda \in C_{7}$.

Lemmas 5.8-5.10 yield the following optimal synthesis for the terminal manifold $S_{6}$.

Corollary 5.11. Let $q_{1} \in S_{6} \backslash\left\{q_{0}\right\}$.

1) If $q_{1} \in S_{i j}, i, j \in\{+,-\}$, then the only optimal trajectory is $\operatorname{Exp}(\lambda, t), t \in\left[0, t_{1}\right]$, where $\left(\lambda, t_{1}\right) \in N_{i j}$ is determined by the equations

$$
x_{1}=i \frac{\sin p}{c}, \quad z_{1}=\frac{2 p-\sin (2 p)}{2 c^{2}}, \quad j p \in(0, \pi), \quad j c \in(0,+\infty) .
$$

2) If $q_{1} \in S_{0 j}, j \in\{+,-\}$, then there are two optimal trajectories $\operatorname{Exp}\left(\lambda_{+}, t\right), \operatorname{Exp}\left(\lambda_{-}, t\right), t \in\left[0, t_{1}\right]$, where $\left(\lambda_{ \pm}, t_{1}\right) \in N_{0 j}^{ \pm}$is determined by the equations

$$
z_{1}=\frac{j \pi}{c^{2}}, \quad \tau= \pm \frac{\pi}{2}
$$

3) If $q_{1} \in S_{i 0}, i \in\{+,-\}$, then the only optimal trajectory is $\operatorname{Exp}(\lambda, t), t \in\left[0, t_{1}\right]$, where $(\lambda, t) \in N_{i 0}$ is determined by the equation $x_{1}=i t_{1}$.

Remark 5.12. If $\operatorname{Exp}\left(\lambda, t_{1}\right) \in S_{6}$ for some $(\lambda, t) \in N$, then $\varepsilon^{6}(\lambda)=\lambda$.

Proof. It follows from Lemmas 5.8-5.10 that the inclusion $\operatorname{Exp}\left(\lambda, t_{1}\right) \in S_{6}$ is possible only in the following two cases:

1) $\lambda \in C_{6}, \tau=\theta+c t / 2= \pm \pi / 2$,

2) $\lambda \in C_{7}, \theta= \pm \pi / 2$.

In both these cases we have $\varepsilon^{6}(\lambda)=\lambda$, since $\varepsilon^{6}:(\theta, c, \alpha,) \mapsto\left(\pi-\theta_{t}, c_{t},-\alpha\right)=\left(\pi-\theta_{t}, c, \alpha\right)$ and $\pi-\theta_{t}=\theta$.

\subsection{Sub-Riemannian sphere}

On the basis of the description of the cut time given by Theorem 5.1, we can study the intersection of the unit sphere $S=\{q \in M \mid d(\mathrm{Id}, q)=1\}$ with the plane $\{x=z=0\}$. One can show that the curve $\gamma=S \cap\{x=z=0\}$ has a decomposition $\gamma=\cup_{i=1}^{4} \gamma_{i} \cup\left\{a_{+}, a_{-}, c_{+}, c_{-}\right\}$, where $\gamma_{i}$ are smooth curves, and $a_{ \pm}, c_{ \pm}$are singular points, near which $\gamma$ is Lipschitzian (see Fig. 4).

The points

$$
c_{ \pm}: x=0, y=0, z=0, w=v-\frac{y^{3}}{6}= \pm \frac{1}{48 K^{2}\left(k_{0}\right)}
$$

are conjugate. The points

$$
a_{ \pm}: x=0, y= \pm 1, z=0, w=v-\frac{y^{3}}{6}=0,
$$

are intersections of the sphere $S$ with abnormal minimizers. Near the point $a_{+}$, the curve $\gamma$ consists of two curves $\gamma_{1}$ and $\gamma_{4}$, where

$$
\gamma_{1}: w=w_{1}(y), \quad y<1,
$$

is a graph of an analytic function, and

$$
\gamma_{4}: w=w_{2}(y), \quad y<1
$$

is a graph of a non-analytic function

$$
w_{2}(y)=-\frac{Y^{3}}{6}+C Y^{3} \mathrm{e}^{-\frac{2}{Y}}(1+o(1)), \quad Y=\frac{1-y}{2}, \quad C=\frac{8}{3}(2-\ln 4)>0 .
$$




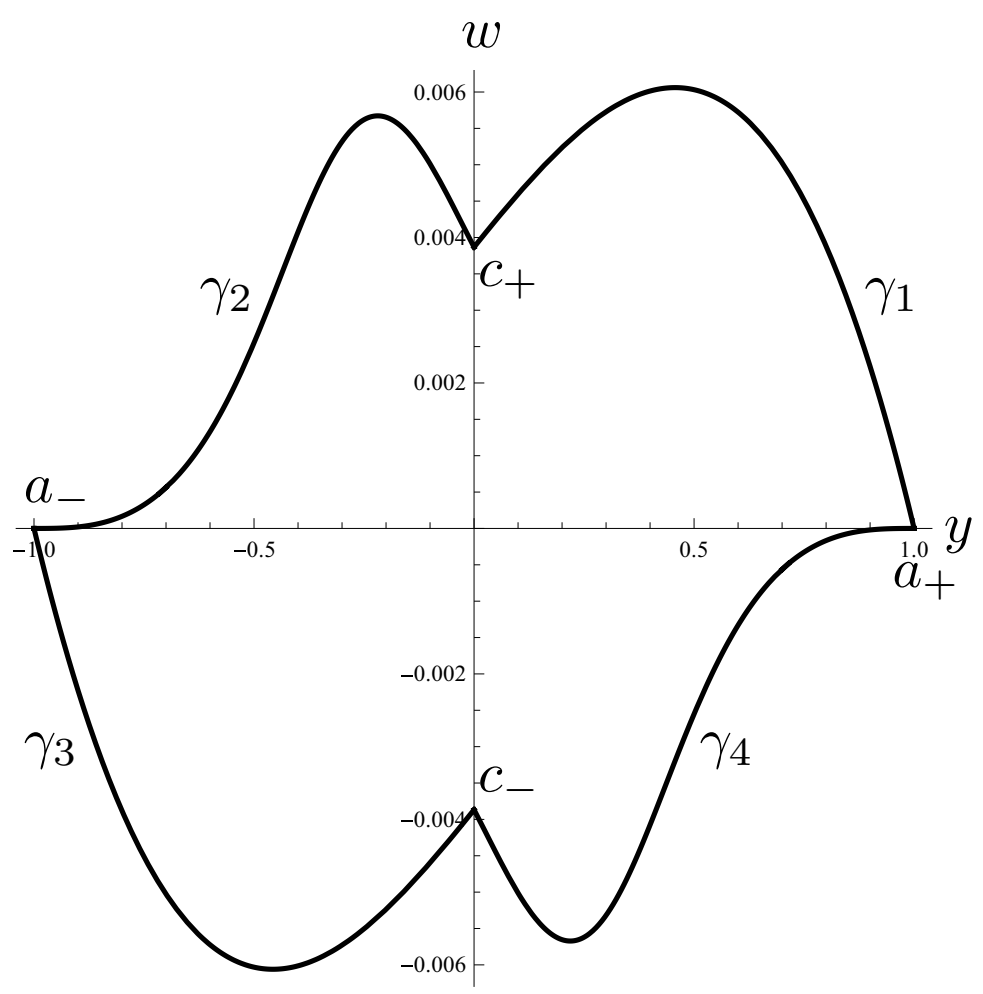

FIGURE 4 . Intersection of the sphere $S$ with the plane $\{x=z=0\}$.

Consequently, the curve $\gamma$ is not semi-analytic. Thus the sphere $S$ is not subanalytic (this fact was previously obtained by B. Bonnard et al. [10] by projecting the SR sphere at the Engel group to the SR sphere in the flat Martinet case). A detailed description of the intersection $\gamma=S \cap\{x=z=0\}$ will be presented elsewhere.

The nilpotent SR geometry on the Engel group exhibits several important features:

(a) existence of abnormal minimizers,

(b) non-subanalyticity of SR spheres,

(c) lack of analytic hypoellipticity of the hypoelliptic Laplacian $\Delta_{H}=X_{1}^{2}+X_{2}^{2}$.

In this problem abnormal geodesics are simultaneously normal, and they project to straight lines in the plane $(x, y)$, see [6]. Since SR length is the length of projections of geodesics to this plane, then abnormal geodesics are minimizers.

Non-subanalyticity of SR spheres on the Engel group was deduced by Bonnard et al. [10] by projecting to the flat Martinet SR geometry. In this work we confirm non-subanalyticity of SR spheres on the Engel group by the study of intersections of the unit sphere with the plane $\{x=z=0\}$.

A differential operator $P$ on a real analytic manifold $M$ is called analytic hypoelliptic if for any real analytic function $f$ on an open set $D \subset M$ all the solutions $u$ to the equation $P u=f$ are real analytic on $D$. It follows from the results of Christ [14] that the hypoelliptic Laplacian $\Delta_{H}=X_{1}^{2}+X_{2}^{2}$ is not analytic hypoelliptic on any Carnot group of step greater or equal to three, with Lie algebra generated by two generators (thus on the Engel group). Notice that a conjecture by Trèves [33] states the equivalence (a) $\Longleftrightarrow$ (c), thus the nilpotent SR geometry on the Engel group supports the Trèves conjecture. 


\section{Conclusion}

We get a description of the global structure of the exponential mapping in the left-invariant sub-Riemannian problem on the Engel group. It was proved that restriction of this mapping to subdomains in the preimage and image of the exponential mapping cut out by the Maxwell strata corresponding to reflections is a diffeomorphism. Thus we reduced the problem to solving a system of algebraic equations. For any terminal point $q_{1}=\left(x_{1}, y_{1}, z_{1}, v_{1}\right)$ with $x_{1} \neq 0$ and $z_{1} \neq 0$ there exists a unique optimal trajectory. Moreover it was proved that the cut time is equal to the first Maxwell time corresponding to reflections.

The cut locus in the sub-Riemannian problem on the Engel group will be described in a forthcoming article. We also plan to study sub-Riemannian spheres and their singularities. Developing software for computation of optimal solutions will allow us to solve the motion planning problem for generic control systems with 4 states and 2 linear inputs via nilpotent approximation (in particular, for the kinematic model of a car with trailer) in the spirit of the works by Gauthier and Zakalyukin [16,17].

Acknowledgements. The authors thank anonymous reviewers for helpful suggestions on improvement of this work.

\section{REFERENCES}

[1] A.A. Agrachev, Geometry of optimal control problems and Hamiltonian systems. In: Nonlinear and Optimal Control Theory, Lect. Notes Math. CIME, 1932. Springer Verlag (2008) 1-59.

[2] A.A. Agrachev and D. Barilari, Sub-Riemannian structures on 3D Lie groups, J. Dyn. Control Syst. 18 (2012) $21-44$.

[3] A.A. Agrachev and Yu.L. Sachkov, Control Theory from the Geometric Viewpoint. Vol. 87 of Encycl. Math. Sci. SpringerVerlag (2004).

[4] A.A. Agrachev, D. Barilari and U. Boscain, Introduction to Riemannian and sub-Riemannian geometry. Lect. Notes. Preprint (2014). Available at https://www.imj-prg.fr/ davide.barilari/Notes.php.

[5] A.A. Agrachev, B. Bonnard, M. Chyba and I. Kupka, Sub-Riemannian sphere in Martinet flat case. ESAIM: COCV 2 (1997) $377-448$.

[6] A.A. Ardentov and Yu.L. Sachkov, Extremal trajectories in nilpotent sub-Riemannian problem on the Engel group. Sbornik: Math. 202 (2011) 1593-1615.

[7] A.A. Ardentov and Yu.L. Sachkov, Conjugate points in nilpotent sub-Riemannian problem on the Engel group. J. Math. Sci. 195 (2013) 369-390.

[8] D.M. Almeida, Sub-Riemannian homogeneous spaces of Engel type. J. Dyn. Control Syst. 20 (2014) $149-166$.

[9] V.N. Berestovskii and I.A. Zubareva, Shapes of spheres of special nonholonomic left-invariant intrinsic metrics on some Lie groups, Siber. Math. J. 42 (2001) 613-628.

[10] B. Bonnard and M. Chyba, Singular Trajectories and their Role in Control Theory. Springer (2003).

[11] U. Boscain and F. Rossi, Invariant Carnot-Caratheodory metrics on $S^{3}$, SO (3), SL (2) and Lens spaces. SIAM J. Control Optim. 47 (2008) 1851-1878.

[12] Y.A. Butt, Yu.L. Sachkov and A.I. Bhatti, Parametrization of extremal trajectories in sub-Riemannian problem on group of motions of pseudo euclidean plane. J. Dyn. Control Syst. 20 (2014) 341-364.

[13] Y.A. Butt, Yu.L. Sachkov and A.I. Bhatti, Maxwell Strata and Conjugate Points in the Sub-Riemannian Problem on the Lie Group $S H(2)$. Preprint arXiv:1408.2043v1 (2014).

[14] M. Christ, Nonexistence of invariant analytic hypoelliptic differential operators on nilpotent groups of step greater than two. Essays on Fourier analysis in honor of Elias M. Stein, Vol. 42 of Princeton Math. Ser. (1995) 127-145.

[15] L. Euler, Methodus inveniendi lineas curvas maximi minimive proprietate gaudentes, sive Solutio problematis isoperimitrici latissimo sensu accepti. Lausanne, Geneva (1744).

[16] J.P. Gauthier and V. Zakalyukin, On the one-step bracket-generating motion planning problem. J. Dyn. Control Syst. 11 (2005) 215-235.

[17] J.P. Gauthier and V. Zakalyukin, On the motion planning problem, complexity, entropy and nonholonomic interpolation. J. Dyn. Control Syst. 12 (2006) 371-404.

[18] V. Jurdjevic, Geometric Control Theory. Cambridge University Press (1997).

[19] S.G. Krantz and H.R. Parks, The Implicit Function Theorem: History, Theory, and Applications. Birkauser (2001).

[20] A.E.H. Love, A Treatise on the Mathematical Theory of Elasticity. New York, Dover (1927).

[21] I. Moiseev and Yu.L. Sachkov, Maxwell strata in sub-Riemannian problem on the group of motions of a plane. ESAIM: COCV 16 (2010) 380-399.

[22] R. Montgomery, A tour of subriemannian geometries, their geodesics and applications. Vol. 94. Math. Surv. Monogr. AMS (2002).

[23] L.S. Pontryagin, V.G. Boltayanskii, R.V. Gamkrelidze and E.F. Mishchenko, The mathematical theory of optimal processes. John Wiley, New York, London (1962). 
[24] Yu.L. Sachkov, Exponential map in the generalized Dido problem. Sb. Math. 194 (2003) 1331-1359.

[25] Yu.L. Sachkov, Discrete symmetries in the generalized Dido problem. Sb. Math. 197 (2006) 235-257.

[26] Yu.L. Sachkov, The Maxwell set in the generalized Dido problem. Sb. Math. 197 (2006) 595-621.

[27] Yu.L. Sachkov, Complete description of the Maxwell strata in the generalized Dido problem. Sb. Math. 197 (2006) 901-950.

[28] Yu.L. Sachkov, Maxwell strata in Euler's elastic problem. J. Dyn. Control Syst. 14 (2008) 169-234.

[29] Yu.L. Sachkov, Conjugate points in Euler's elastic problem. J. Dyn. Control Syst. Springer, New York (2008) $14409-439$.

[30] Yu.L. Sachkov, Conjugate and cut time in sub-Riemannian problem on the group of motions of a plane. ESAIM: COCV 16 (2010) 1018-1039.

[31] Yu.L. Sachkov, Cut locus and optimal synthesis in the sub-Riemannian problem on the group of motions of a plane. ESAIM: COCV 17 (2011) 293-321.

[32] Yu.L. Sachkov and E.F. Sachkova, Exponential mapping in Euler's elastic problem. J. Dyn. Control Syst. 20 (2014) $443-464$.

[33] F. Trèves, Analytic hypo-ellipticity of a class of pseudodifferential operators with double characteristics and applications to the $\bar{\partial}$-Neumann problem. Commun. Partial Differ. Equ. 3 (1978) 475-642.

[34] A.M. Vershik and V.Ya. Gershkovich, Nonholonomic dynamical systems, geometry of distributions and variational problems. Dynamical Systems VII. Encycl. Math. Sci. (1990) 4-79.

[35] E.T. Whittaker and G.N. Watson, A Course of Modern Analysis. Cambridge University Press (1927). 